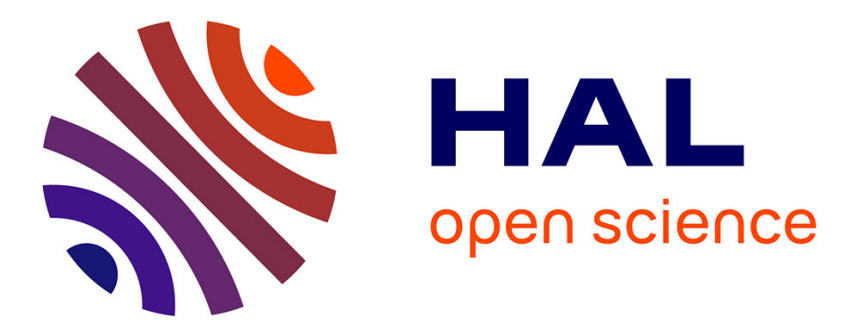

\title{
Switching, relay and complementarity systems: A tutorial on their well-posedness and relationships
}

Carmina Georgescu, Bernard Brogliato, Vincent Acary

\section{To cite this version:}

Carmina Georgescu, Bernard Brogliato, Vincent Acary. Switching, relay and complementarity systems: A tutorial on their well-posedness and relationships. Physica D: Nonlinear Phenomena, 2012, Dynamics and Bifurcations of Nonsmooth Systems, 241 (22), pp.50. 10.1016/j.physd.2011.10.014 . hal-00646982

\section{HAL Id: hal-00646982 \\ https://hal.inria.fr/hal-00646982}

Submitted on 1 Feb 2013

HAL is a multi-disciplinary open access archive for the deposit and dissemination of scientific research documents, whether they are published or not. The documents may come from teaching and research institutions in France or abroad, or from public or private research centers.
L'archive ouverte pluridisciplinaire HAL, est destinée au dépôt et à la diffusion de documents scientifiques de niveau recherche, publiés ou non, émanant des établissements d'enseignement et de recherche français ou étrangers, des laboratoires publics ou privés. 
INSTITUT NATIONAL DE RECHERCHE EN INFORMATIQUE ET EN AUTOMATIQUE

\title{
Switching, relay and complementarity systems: a tutorial on their well-posedness and relationships
}

\author{
Carmina Georgescu - Bernard Brogliato - Vincent Acary
}

\section{$\mathbf{N}^{\circ} \mathbf{7 7 6 0}$}

Septembre 2011

Modeling, Optimization, and Control of Dynamic Systems

\section{apport}

de recherche 


\title{
Switching, relay and complementarity systems: a tutorial on their well-posedness and relationships
}

\author{
Carmina Georgescu * ${ }^{*}$ Bernard Brogliato ${ }^{\dagger}$, Vincent Acary ${ }^{\ddagger}$ \\ Theme : Modeling, Optimization, and Control of Dynamic Systems \\ Applied Mathematics, Computation and Simulation \\ Équipes-Projets Bipop
}

Rapport de recherche $n^{\circ} 7760$ - Septembre 2011 - 50 pages

\begin{abstract}
In this work we focus on analyzing the relationships between switching systems defined from a partition of the state space into convex cells, and relay or complementarity dynamical systems, which are other classes of discontinuous systems. First the conditions guaranteing the continuity of the vector field of the switching system at the cells boundaries (in which case the switching system is an ordinary differential equation with Lipschitz right-hand-side) are recalled. Then well-posedness results (i.e. results on the existence and the uniqueness of solutions) for different classes of relay and complementarity systems which are also switching systems are reviewed. The reverse issue (when can a switching system be rewritten equivalently as a relay or a complementarity system) is also tackled. Many examples from Mechanics, Circuits, Biology, illustrate the developments all through the paper. The paper focuses on systems with continuous solutions (i.e. with no state jumps). Convexity is the central property.
\end{abstract}

Key-words: discontinuous systems, relay systems, well-posedness, complementarity systems, piecewise linear systems, dissipative systems, maximal monotone operators, Lur'e systems, multivalued systems, differential inclusions, Filippov's systems.

\footnotetext{
* University Politehnica of Bucharest, Faculty of Applied Sciences, Spl. Independent, ei, 313, 060042, Bucharest, Romania; Email: carmina amd@yahoo.com

${ }^{\dagger}$ INRIA, BIPOP team-project, Inovallée, 655 avenue de l'Europe, 38330, Montbonnot, France

$\ddagger$ INRIA, BIPOP team-project, Inovallée, 655 avenue de l'Europe, 38330, Montbonnot, France
} 


\section{Systèmes à commutations, à relais et systèmes de complémentarité: existence et unicité des solutions, et équivalences}

Résumé : Dans cet article nous nous concentrons sur l'analyse des relations entre les systèmes à commutations définis à partir d'une partition de l'espace d'état en cellules convexes, les systèmes à relais et les systèmes de complémentarité. En premier lieu les conditions garantissant la continuité du champ de vecteur sur le bord des cellules (auquel cas le système est une ODE avec second membre Lipschitz) sont rappelées. Ensuite des résultats d'existence et unicité concernant diverses classes de systèmes à relais et de complémentarité qui sont des systèmes à commutations sont passés en revue. Le problème inverse (quand est-ce qu'un système à commutation peut être représenté comme un système à relais ou de complémentarité) est abordé aussi. De nombreux examples de la mécanique, des circuits, de la biologie, illustrent les développements. Le cas des solutions discontinues n'est pas abordé. La convexité apparait comme la propriété centrale de ces systèmes.

Mots-clés : systèmes discontinus, systèmes à relais, existence et unicité de solutions, systèmes de complémentarité, systèmes dissipatifs, opérateurs maximaux monotones, systèmes de Lur'e, inclusions différentielles, systèmes de Filippov. 


\section{Introduction}

Discontinuous systems, i.e. dynamical systems whose right-hand side is not a continuous vector field, have become very popular and much studied in various scientific fields like Applied Mathematics [40, 34], Systems and Control [60, 26], Mechanics [11, 42], Biology [23, 33, 43], Electricity and Electronics [53, 3, 4, 41], etc. They form such a huge class of systems (just like nonlinear systems do) that it is mandatory to consider particular subclasses of nonsmooth systems in order to analyze them. A survey of various mathematical formalisms for nonsmooth systems may be found in [2, Chapter $2]$ and [3, Chapter 2]. In this paper we consider systems which are defined from the subdivision of the ambient state space $\mathbb{R}^{n}$ into cells, and with each cell is associated a smooth vector field. The trajectories may travel from one cell to the other, possibly implying jumps in the system's right-hand side. Such systems are usually called switching systems. Despite their apparent simplicity, they are strongly nonlinear and nonsmooth (i.e. most often their nonlinearity and nonsmoothness cannot be removed by any suitable state vector change or by feedback). Our objective is mainly to provide an overview of the results that allow to state the existence and uniqueness of solutions, and this can be done only for specific cases of switching systems. This paper also provides information on the relationships between various mathematical formalisms (switching systems, relay systems, complementarity systems, Filippov's differential inclusions), an objective that may be useful for a better understanding of such nonsmooth dynamical systems, as advocated in $[12,13,14,16]$. There are two issues which are tackled in this survey: when do relay and complementarity systems belong to the class of switching systems? When can switching systems be represented as relay or complementarity systems? The objective in both studies remains the same: find subclasses of switching systems that lend themselves well to mathematical analysis (especially uniqueness of solutions issues), numerical analysis and simulation.

Paper organization. The remainder of the article is organized as follows. In Section 2 we list some definitions and notations which are used throughout the paper. In Section 3 we display the switching systems framework, while in Section 4 conditions for the existence of classical solutions are discussed. The method based on the Filippov framework is described in Section 5. Different classes of dynamical systems as well as interconnections with the general class of switching systems are largely investigated in Sections 6-10, 12-13. Finally, Section 15 concludes the paper with pointers to the sections where all these dynamical systems and the relationships between them have been analyzed (see Figure 1).

\section{Basic notations and definitions.}

All the notions cited below can be found in $[27,38,66]$. For $x \in \mathbb{R}^{n}$, we write $x \succ 0$ if

$$
x_{1}>0 \quad \text { or } \quad\left[x_{i}=0, i \in \overline{1, i_{0}} \text { and } x_{i_{0}+1}>0\right]
$$

for some $i_{0} \in \overline{1, n-1}$. If $x=0$ or $x \succ 0$ we denote it $x \succeq 0$. The script $\|$.\| will stand for the Euclidean norm in $\mathbb{R}^{n}$ and (., .) will denote the inner product. The extended $L^{p}$ space is denoted by $L_{l o c}^{p}$. 


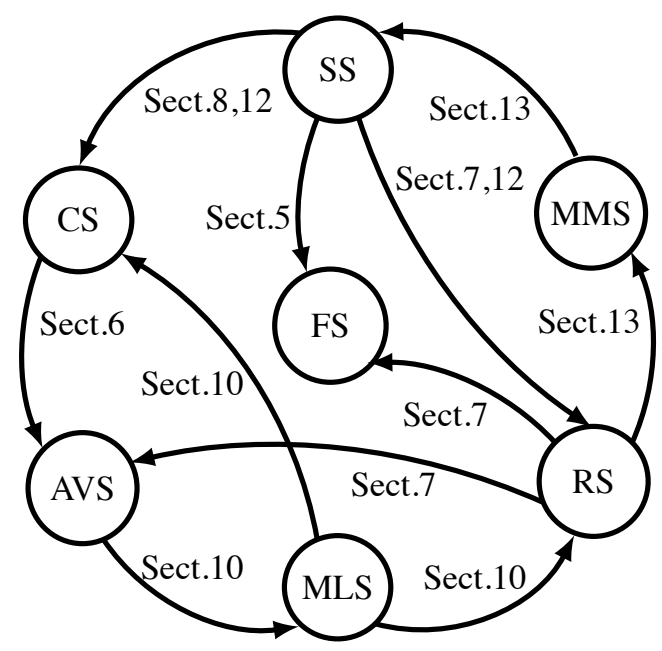

Figure 1: Relationships between different classes of dynamical systems. SS=switching systems; MMS=multimodal systems; RS=relay systems; AVS= affine variational systems; MLS=multivalued Lur'e systems; CS=complementarity systems; FS=Filippov systems.

We recall that given $f: \mathbb{R}^{n} \rightarrow \mathbb{R}^{n}$ and $h: \mathbb{R}^{n} \rightarrow \mathbb{R}$ two smooth functions, the Lie derivative of order $k \in \mathbb{N}$ of $h$ along $f$ is given by $L_{f}^{k} h: \mathbb{R}^{n} \rightarrow \mathbb{R}$,

$$
L_{f}^{k} h(x)=\left\{\begin{array}{cl}
h(x) & \text { if } \quad k=0 \\
\left(\frac{\partial}{\partial x} L_{f}^{k-1} h(x)\right) f(x) & \text { if } \quad k \geq 1
\end{array} .\right.
$$

A switching system is said to have an accumulation point $\tau \geq 0$ of switches at the right of $\tau$ if for any switched point $T>\tau$, there exists another one $T^{\prime}>\tau$ such that $T^{\prime}<T$ and the sequence of these switches tends to $\tau$. An accumulation point of switches at the left of $\tau$ is defined similarly by taking opposite inequalities in the above definition.

A matrix $M \in \mathcal{M}_{m, m}(\mathbb{R})$ is said to be a $P$-matrix if all its principal minors are strictly positive. $M$ is said to be a $P_{0}$-matrix if all its principal minors are nonnegative. $M_{\bullet j}$ is its $j$ th column, and $M_{i \bullet}$ is its $i$ th row. The closure of a set $\chi \subset \mathbb{R}^{n}$ is denoted $\bar{\chi} . B(x, r)$ denotes the closed ball of $\mathbb{R}^{n}$ centered at $x \in \mathbb{R}^{n}$ of radius $r$.

For a convex set $S$, the normal cone to $S$ at $x \in S$ is the set $\{y:\langle v-x, y\rangle \leq$ $0, \forall v \in S\}$. For any $K \subset \mathbb{R}^{n}$, the indicator function $\psi_{K}$ of the set $K$ is given by: $\psi_{K}(\lambda)=0$ if $\lambda \in K$, and $\psi_{K}(\lambda)=+\infty$ if $\lambda \notin K$. The convex closure of a set $A \subset \mathbb{R}^{n}$ is denoted by $\overline{c o}(A)$.

A (possibly multivalued) operator $F$ is said to be monotone if for any $x_{1} \in \operatorname{Dom}(F)$, $x_{2} \in \operatorname{Dom}(F), y_{1} \in F\left(x_{1}\right), y_{2} \in F\left(x_{2}\right)$, one has $\left\langle x_{1}-x_{2}, y_{1}-y_{2}\right\rangle \geq 0$ where $\operatorname{Dom}(F)=\{x: F(x) \neq \emptyset\}$. It is maximal if its graph cannot be enlarged without destroying the monotonicity.

Let $X$ and $Y$ be two topological spaces. A multifunction $G: X \rightarrow \mathcal{P}(Y)$ is said to be upper semicontinuous (u.s.c.) at $x \in X$ if whenever $V$ is an open subset of $Y$ that contains $G(x)$, the set $\{x: G(x) \subset V\}$ contains a neighborhood of $x$. It is called 
u.s.c. on $\mathrm{X}$, if it is u.s.c. at each $x \in X$. We say that $F: \mathbb{R}^{n} \rightarrow \mathcal{P}\left(\mathbb{R}^{p}\right)$ is one-sided Lipschitz continuous if there exists $\lambda \in \mathbb{R}$ such that

$$
\left(y^{\prime}-y^{\prime \prime}, x^{\prime}-x^{\prime \prime}\right) \leq \lambda\left\|x^{\prime}-x^{\prime \prime}\right\|^{2},
$$

for all $y^{\prime} \in F\left(x^{\prime}\right), y^{\prime \prime} \in F\left(x^{\prime \prime}\right)$, with $x^{\prime}, x^{\prime \prime} \in \operatorname{Dom}(F)$.

A function $f$ is said to be proper if $\operatorname{dom}(f)=\{x: f(x)<+\infty\}$ is non-empty and the restriction of $f$ to $\operatorname{dom}(f)$ is finite. If $f$ is a proper convex function, its conjugate is the proper convex function

$$
f^{*}: z \mapsto \sup _{x \in \operatorname{dom}(f)}(\langle x, z\rangle-f(x)) .
$$

\section{The class of switching systems}

In this section we provide the description of a class of discontinuous systems, usually known as switching systems $[34,50]$.

\subsection{Description of the cells}

Let $d_{j}: \mathbb{R}^{n} \rightarrow \mathbb{R}, d_{j}(x)=H_{j \bullet} x+h_{j}$ with $j \in \overline{1, p}$ where $H \in \mathcal{M}_{p, n}(\mathbb{R})$, and $h_{j} \in \mathbb{R}$. It is well known that the intersection of half-spaces defined by hyperplanes is a closed convex polyhedron. In what follows we consider that the state space $\mathbb{R}^{n}$ is split into $m\left(m \leq 2^{p}\right)$ open polyhedral cells $\left(\chi_{i}\right)_{i \in \overline{1, m}}$, with pairwise disjoint interior, each of them having the following redundant description

$$
x \in \chi_{i} \text { if and only if }\left\{\begin{array}{r}
d_{j}(x)>0, \quad \forall j \in J_{i}^{1} \\
-d_{j}(x)>0, \quad \forall j \in J_{i}^{2},
\end{array}\right.
$$

where $J_{i}^{1} \cup J_{i}^{2}=\overline{1, p}$ and $J_{i}^{1} \cap J_{i}^{2}=\emptyset$. For $j \in \overline{1, p}$, let $\Sigma_{j}=\left\{x \in \mathbb{R}^{n}: d_{j}(x)=0\right\}$; so the sets $\chi_{i}, i \in \overline{1, m}$ are separated by codimension one surfaces (switching surfaces) and $\cup_{i \in \overline{1, m}} \overline{\chi_{i}}=\mathbb{R}^{n}$. The expression "redundant" description refers to the fact that even if one inequality may imply another one, we agree to describe each cell by the help of all $p$ surfaces in order to have a systematic description.

Example 3.1. Consider in the plane $x_{1} O x_{2}$ a family of parallel lines $\left\{d_{j}: j \in \overline{1, p}\right\}$ given by

$$
d_{j}: H_{j, 1} x_{1}+H_{j, 2} x_{2}+h_{j}=0
$$

such that $H_{j, 1}<0 \forall j \in \overline{1, p}$ and $\frac{h_{1}}{H_{1,1}}>\frac{h_{2}}{H_{2,1}}>\ldots>\frac{h_{p}}{H_{p, 1}}$. In accordance with the above description of the cells (here $m=p+1$ ), we adopt the following

$$
\begin{array}{ll}
\chi_{1}: & d_{1}(x)>0, d_{2}(x)>0, \ldots, d_{p}(x)>0 ; J_{1}^{1}=\overline{1, p}, J_{1}^{2}=\emptyset \\
\chi_{2}: & d_{1}(x)<0, d_{2}(x)>0, \ldots, d_{p}(x)>0 ; J_{2}^{1}=\overline{2, p}, J_{2}^{2}=\{1\} \\
\vdots & \\
\chi_{p+1}: & d_{1}(x)<0, \ldots, d_{p}(x)<0 ; J_{p+1}^{1}=\emptyset, J_{p+1}^{2}=\overline{1, p} .
\end{array}
$$

The preference for the above redundant description finds explanation in Section 13 when trying to identify a class of discontinuous systems that can be analyzed with the help of piecewise-linear systems as well as using the theory of relay inclusions. This may be viewed as an attempt to partially fill the gap between these two formalisms widely employed in the theory of switching systems. 


\subsection{General formulation of the dynamics}

In this paper we focus on dynamical systems ([10], [50]) modelled by the following autonomous Initial Value Problem (IVP on short):

$$
\left\{\begin{aligned}
\dot{x}(t) & =f(x(t)), \quad f(x(t))=f_{i}(x(t)) \text { if } x(t) \in \chi_{i}, i \in \overline{1, m} \\
x\left(t_{0}\right) & =x_{0} \in \mathbb{R}^{n},
\end{aligned}\right.
$$

where $f_{i}: \bar{\chi}_{i} \rightarrow \mathbb{R}^{n}, i \in \overline{1, m}$ is a smooth function in $\operatorname{Int}\left(\chi_{i}\right)$, the interior of the set $\chi_{i}$. The system is completely described by (2) outside the discontinuity boundaries, where two or more different vectors can be associated to a point $x \in \Sigma_{j}$. Without loss of generality, the initial time can be taken as $t_{0}=0$.

This definition of switching dynamical systems is in fact only a preliminary definition. The analysis and the numerical simulation of such systems will most of the time require to add more information in the model, and to drastically narrow, for instance, the topology of the division of $\mathbb{R}^{n}$ into cells. It is noteworthy that we focus only on the switching systems with time-continuous trajectories. Systems with state jump are outside the scope of this paper.

Example 3.2. Let us consider a mass $m$ that collides an obstacle modelled as a linear spring/dashpot system with stiffness $k>0$ and damping $c>0$. The coordinate of the mass is $q$, the state is $x^{T}=(q, \dot{q})$. The dynamics is:

$$
m \ddot{q}(t)= \begin{cases}-k q(t)-c \dot{q}(t) & \text { if } q>0 \\ 0 & \text { if } q<0\end{cases}
$$

An important assumption in (3) is that the switch between contact/non contact phases is done instantaneously when q passes through 0 . From the mechanical point of view this may not be satisfying as it is known that such a model may create contact forces with the wrong sign (as if the obstacle would attract the mass instead of always pushing it [5]). A better way to model the switch will be presented in Example 8.1. It is noteworthy that since the right-hand side of (3) may be discontinuous, the classical results for the existence and uniqueness of solutions do not hold. Here we have: $p=1, d_{1}(x)=q$, $J_{1}^{1}=\{1\}, J_{1}^{2}=\emptyset$. Other mass/spring/dashpot systems are considered in [49].

\section{Continuity of $f$ at the boundaries}

Obviously, when the right-hand side in (2) is continuous, the existence of classical solutions (continuously differentiable or, on short, $\mathcal{C}^{1}$ functions) is ensured by the wellknown Peano's Theorem for ordinary differential equations. The discussion upon the continuity of dynamical system (2) could be useful for several reasons. For example, if the system (2) is continuous, then it can be simulated by using classical numerical methods, while if it is discontinuous, only special numerical methods should be used [2]. In this section we are concerned with a particular class of dynamical systems modelled by (2) namely,

$$
\dot{x}=A_{i} x+a_{i} \text { if } x \in \chi_{i},
$$

where $A_{i} \in \mathcal{M}_{n, n}(\mathbb{R})$ is a constant matrix and $a_{i} \in \mathbb{R}^{n}$ is a constant vector. The dynamics in (4) defines a piecewise affine system. In general, if no additional conditions are stated on the terms $A_{i}, a_{i}, H_{j}$, and $h_{j}$, the right-hand-side of (4) is discontinuous at the intersection boundaries $\partial \chi_{i}$. 
The continuity of $f$ at the intersection boundary $\Sigma_{1,2} \subseteq\left\{x \in \mathbb{R}^{n}: H_{j \bullet} x+h_{j}=\right.$ $0\}$ between two regions $\bar{\chi}_{i_{1}}$ and $\bar{\chi}_{i_{2}}$ holds if and only if $\Sigma_{1,2} \subseteq \operatorname{Ker}\left(\mathcal{A}_{i_{1}}-\mathcal{A}_{i_{2}}\right)$, where the operators $\mathcal{A}_{i_{k}}: x \mapsto A_{i_{k}} x+a_{i_{k}}, k=1,2$, and $\operatorname{Ker}\left(\mathcal{A}_{i_{1}}-\mathcal{A}_{i_{2}}\right)=\left\{x \in \mathbb{R}^{n}:\right.$ $\left.A_{i_{1}} x+a_{i_{1}}-A_{i_{2}} x-a_{i_{2}}=0\right\}$. In the next theorem we show that this condition has to be fulfilled on all codimension 1 boundaries. At the codimension $>1$ boundaries, the continuity of the system is obtained immediately from the continuity condition between two any cells separated by a hyperplane.

Theorem 4.1. The right-hand side of the system (4) is continuous if and only if the following condition is satisfied: for any two cells $\chi_{i_{1}}$ and $\chi_{i_{2}}$ separated by a hyperplane $\left\{x \in \mathbb{R}^{n}: H_{j} \bullet x+h_{j}=0\right\}$, one has

$$
\left\{x \in \mathbb{R}^{n}: H_{j \bullet} x+h_{j}=0\right\} \subseteq \operatorname{Ker}\left(\mathcal{A}_{i_{1}}-\mathcal{A}_{i_{2}}\right) .
$$

Proof. We prove that $\mathcal{A}_{i_{1}}(x)=\mathcal{A}_{i_{2}}(x)$ on $\left\{x \in \mathbb{R}^{n}: H_{j} \bullet x+h_{j}=0\right\}$ if and only if $\mathcal{A}_{i_{1}}(x)=\mathcal{A}_{i_{2}}(x)$ on $D \cap\left\{x \in \mathbb{R}^{n}: H_{j \bullet} x+h_{j}=0\right\}$, where $D$ is an open subset of $\mathbb{R}^{n}$ such that $D \cap\left\{x \in \mathbb{R}^{n}: H_{j \bullet} x+h_{j}=0\right\} \neq \emptyset$.

Since the necessity is obvious, let us prove the sufficiency. We suppose that $\mathcal{A}_{i_{1}}(x)=$ $\mathcal{A}_{i_{2}}(x)$ on $D \cap\left\{x \in \mathbb{R}^{n}: H_{j} \bullet x+h_{j}=0\right\}$ and prove that this equality remains valid on the whole set $\left\{x \in \mathbb{R}^{n}: H_{j \bullet} x+h_{j}=0\right\}$. Let $v \in D \cap\left\{x \in \mathbb{R}^{n}: H_{j} \bullet x+h_{j}=\right.$ $0\} \neq \emptyset$ and $r>0$ such that $B(v, r) \subseteq D$. So $\mathcal{A}_{i_{1}}(v)=\mathcal{A}_{i_{2}}(v)$. We know that for any hyperplane $H \subseteq \mathbb{R}^{n}$ and $v \in H$ there exists a subspace $S \subseteq \mathbb{R}^{n}$ with $\operatorname{dim}(S)=n-1$ such that $H=v+S$. Consider a base $\left\{v_{1}, v_{2}, \ldots, v_{n-1}\right\}$ in $S$. Then, for any $x$ with $H_{j} \bullet x+h_{j}=0$ there exist $\lambda_{1}, \ldots, \lambda_{n-1} \in \mathbb{R}$ such that $x=v+\sum_{k=1}^{n-1} \lambda_{k} v_{k}$. We have

$$
\mathcal{A}_{i_{1}}(x)=\mathcal{A}_{i_{2}}(x) \Leftrightarrow \sum_{k=1}^{n-1} \lambda_{k} A_{i_{1}} v_{k}=\sum_{k=1}^{n-1} \lambda_{k} A_{i_{2}} v_{k} .
$$

Further, for all $k \in \overline{1, n-1}$, define $w_{k}=v+\frac{r}{2\left\|v_{k}\right\|} v_{k}$. It is clear that $w_{k} \in B(v, r) \cap$ $\left\{x \in \mathbb{R}^{n}: H_{j \bullet} x+h_{j}=0\right\}$ and therefore, $\mathcal{A}_{i_{1}}\left(w_{k}\right)=\mathcal{A}_{i_{2}}\left(w_{k}\right)$ which implies that $A_{i_{1}} v_{k}=A_{i_{2}} v_{k}$. From (5), we conclude that $\mathcal{A}_{i_{1}}(x)=\mathcal{A}_{i_{2}}(x)$ for any $x \in \mathbb{R}^{n}$, verifying $H_{j} \bullet x+h_{j}=0$.

Remark 4.2. In fact, Theorem 4.1 says that a linear function on $\mathbb{R}^{n}$ vanishes on a hyperplane $\mathcal{H} \subseteq \mathbb{R}^{n}$ if and only if it vanishes on the intersection of $\mathcal{H}$ with an open set in $\mathbb{R}^{n}$. More generally, if $b_{1}, c_{1} ; \ldots ; b_{n-1}, c_{n-1} \in \mathcal{H}$ are such that the vectors $b_{1}-c_{1}, \ldots$, $b_{n-1}-c_{n-1}$ are linear independent, a linear function vanishes on the whole hyperplane $\mathcal{H}$ if and only if it vanishes at each $b_{1}, c_{1} ; \ldots ; b_{n-1}, c_{n-1}$ (see Example 4.3 below). This still remains valid if we replace the hyperplane in $\mathbb{R}^{n}$ by an affine manifold (here, by an affine manifold we mean a linear subspace possibly shifted away from the origin) of dimension $d \leq n-1$. We recall that a hyperplane is an affine manifold of dimension $d=n-1$. In $\mathbb{R}^{n}$, the hyperplanes describe tangent planes to a smooth hypersurface.

Example 4.3. In $\mathbb{R}^{3}$ the hyperplanes $x_{1}=0, x_{2}=0$ and $x_{3}=0$ determine eight cells. Let us consider two regions

$$
\begin{aligned}
& \chi_{1}: x_{1}>0, x_{2}>0, \quad x_{3}>0 \\
& \chi_{2}: x_{1}>0, x_{2}>0,-x_{3}>0
\end{aligned}
$$

separated by the hyperplane $x_{3}=0$. In these cells we suppose that the system (4) is defined such that for $\chi_{1}$ and $\chi_{2}$ we have: $A_{1}-A_{2}=\left(\begin{array}{ccc}0 & 1 & 0 \\ 0 & -1 & 0 \\ 0 & 2 & 0\end{array}\right)$ and $a_{1}=a_{2}$. We 
notice that $\mathcal{A}_{1}(x)=\mathcal{A}_{2}(x)$ for all $x=\left(x_{1}, 0, x_{3}\right)^{T}$ with $x_{1}, x_{3} \in \mathbb{R}$, so $\mathcal{A}_{1}-\mathcal{A}_{2}$ vanishes on a whole line in the hyperplane $x_{3}=0$, in particular at $e_{1}=(1,0,0)^{T}$ but not at $e_{2}=(0,1,0)^{T}$, hence no matter how the system is defined in the other cells, the global continuity of $f$ fails.

The following theorem establishes necessary and sufficient conditions for the continuity of the right-hand side of (4). We include here the proof, slightly different from the one given in [63], which allows one to formulate an algorithm in order to investigate system (4) for the existence of classical solutions. The proof uses a priori the previous result contained in Theorem 4.1.

Theorem 4.4. [63] Consider system (4). The right-hand side of system (4) is continuous if and only if the following condition is satisfied: for any two cells $\chi_{i_{1}}$ and $\chi_{i_{2}}$ having a common boundary $H_{j \bullet} x+h_{j}=0$, there exists $v_{1,2} \in \mathcal{M}_{n, 1}(\mathbb{R})$ such that the corresponding matrices $A_{i_{1}}$ and $A_{i_{2}}$ and the vectors $a_{i_{1}}$ and $a_{i_{2}}$ satisfy the equalities

$$
\begin{gathered}
v_{1,2} H_{j \bullet}=A_{i_{1}}-A_{i_{2}} \\
v_{1,2} h_{j}=a_{i_{1}}-a_{i_{2}} .
\end{gathered}
$$

Proof. The sufficiency is obvious. To check the continuity we notice that, for any $x$ such that $d_{j}(x)=0$, we have

$$
v_{1,2}\left(H_{j} x+h_{j}\right)=\left(A_{i_{1}}-A_{i_{2}}\right) x+a_{i_{1}}-a_{i_{2}}=0
$$

so $f($.$) is continuous on the boundary. In order to prove the necessity, we shall consider$ two cases.

Case $1 . h_{j}=0$. Then, a compatibility condition for the continuity of $f($.$) must$ hold: $a_{i_{1}}=a_{i_{2}}$. This is readily seen, since $f($.$) is continuous at any point on the$ boundary, in particular at $x=0$. In this case, the second equality in the conclusion of the theorem is proved.

Next, from the continuity of $f($.$) , it results that \left(A_{i_{1}}-A_{i_{2}}\right) x=0$ for all $x \in \mathbb{R}^{n}$ with $H_{j} . x=0$. Let us denote by $\left(A_{i_{1}}-A_{i_{2}}\right)_{r}$ the r-th line of matrix $A_{i_{1}}-A_{i_{2}}$. So $\left(A_{i_{1}}-A_{i_{2}}\right)_{r} \in\left(\operatorname{Ker}\left(H_{j} .\right)\right)^{\perp}$ which is spanned by $H_{j}$. Form this we deduce that $\left(A_{i_{1}}-A_{i_{2}}\right)_{r}$ and $H_{j}$. are collinear, that is there exists $\mu_{r} \in \mathbb{R}$ such that $\left(A_{i_{1}}-A_{i_{2}}\right)_{r}=$ $\mu_{r} H_{j}$. Putting $v_{1,2}=\left(\mu_{1}, \ldots, \mu_{n}\right)^{T}$, we get equality (6) in the conclusion of the theorem.

Case 2. $h_{j} \neq 0$. We prove that for any $\tilde{x} \in \operatorname{Ker}\left(H_{j}.\right)$ we have $\left(A_{i_{1}}-A_{i_{2}}\right) x=$ 0 . Indeed, for all $x \in \mathbb{R}^{n}$ verifying $H_{j} x+h_{j}=0$ and $\tilde{x} \in \operatorname{Ker}\left(H_{j}\right.$. $)$ we have $H_{j .}(x-\tilde{x})+h_{j}=0$. From the continuity of $f($.$) at x$ and $\tilde{x}$, we get $A_{i_{1}}(x)=A_{i_{2}}(x)$. Next, using similar arguments as in Case 1 , we obtain the existence of a vector $v_{1,2}$ such that $v_{1,2} H_{j .}=A_{i_{1}}-A_{i_{2}}$.

It remains to show the second equality. To this purpose, we invoke again the continuity of $f($.$) and replacing A_{i_{1}}-A_{i_{2}}$ by $v_{1,2} H_{j}$, we may write

$$
v_{1,2} H_{j} x+a_{i_{1}}-a_{i_{2}}=0 \quad \forall x \text { with } H_{j .} x+h_{j}=0
$$

and so $v_{1,2} h_{j}=a_{i_{1}}-a_{i_{2}}$.

Remark 4.5. We point out that if $a_{i_{1}}=a_{i_{2}}$ (in particular null vectors) and $A_{i_{1}} \neq A_{i_{2}}$ then, in order for $f$ to be continuous, we must have $h_{j}=0$. This condition is only 
necessary. There are cases when $h_{j}=0$, but the vector field jumps (see for instance (3)). This is explained by the fact that, in general, the condition $h_{j}=0$ does not guarantee the collinearity between all the lines of the matrix $A_{i_{1}}-A_{i_{2}}$ and the vector $H_{j}$. If, in addition, this condition is satisfied, then the vector field is continuous.

Remark 4.6. From the continuity property it follows that, for any point on the boundary $\Sigma_{j}$ between any two cells $\chi_{i_{1}}$ and $\chi_{i_{2}}$, we must have $a_{i_{2}}-a_{i_{1}}=\left(A_{i_{1}}-A_{i_{2}}\right) x \subset$ $\operatorname{Im}\left(A_{i_{1}}-A_{i_{2}}\right)$. So, in order for (4) to be a continuous system, it should verify the following consistency condition:

$$
a_{i_{2}}-a_{i_{1}} \in \operatorname{Im}\left(A_{i_{1}}-A_{i_{2}}\right) .
$$

In the next theorem we give other equivalent conditions for the continuity of system (4) in terms of the kernels. For the sake of simplicity, let us denote $A_{1,2}=A_{1}-A_{2}$ and $a_{1,2}=a_{1}-a_{2}$.

Theorem 4.7. The right-hand side of system (4) is continuous if and only if for any two cells $\chi_{i_{1}}$ and $\chi_{i_{2}}$ having a common boundary $H_{j} \bullet+h_{j}=0$, the vector fields corresponding to these cells satisfy:

$$
\begin{gathered}
A_{1,2} H_{j \bullet}^{+} h_{j}=a_{1,2} \\
\operatorname{Ker}\left(H_{j \bullet}\right) \subseteq \operatorname{Ker}\left(A_{1,2}\right),
\end{gathered}
$$

where $H_{j \bullet}^{+}=H_{j \bullet}^{T}\left(H_{j \bullet} H_{j \bullet}^{T}\right)^{-1}$ stands for the Moore-Penrose pseudoinverse of $H_{j \bullet}$.

Proof. By virtue of Theorem 4.1, we know that the global continuity of $f$ holds if and only if for any two cells $\chi_{i_{1}}$ and $\chi_{i_{2}}$ having a common boundary $H_{j} \bullet x+h_{j}=0$, one has $A_{1,2} x+a_{1,2}=0$ for all $x$ with $H_{j} \bullet x+h_{j}=0$, that is

$$
A_{1,2}\left(-H_{j \bullet}^{+} h_{j}+\alpha\right)+a_{1,2}=0 \quad \forall \alpha \in \operatorname{Ker}\left(H_{j \bullet}\right) .
$$

Further, this equality is equivalent to the following ones

$$
\begin{gathered}
A_{1,2} H_{j \bullet}^{+} h_{j}=a_{1,2} \\
\operatorname{Ker}\left(H_{j \bullet}\right) \subseteq \operatorname{Ker}\left(A_{1,2}\right),
\end{gathered}
$$

that completes the proof.

Remark 4.8. $i$ ) If $A_{i_{1}} \neq A_{i_{2}}$, then $\operatorname{rank}\left(A_{1,2}\right) \geq 1$ and from Theorem 4.7, we deduce that

$$
\operatorname{dim}\left(\operatorname{Ker}\left(A_{1,2}\right)\right)=\operatorname{dim}\left(\operatorname{Ker}\left(H_{j} \bullet\right)\right)=n-1 .
$$

ii) Considering Example 3.2, we notice that for $c \neq 0$,

$$
\begin{aligned}
\operatorname{KerH} & =\left\{x=\left(x_{1}, x_{2}\right)^{T}: x_{1}=0\right\} \\
\not \operatorname{Ker} A & =\left\{x=\left(x_{1}, x_{2}\right)^{T}: k x_{1}+c x_{2}=0\right\},
\end{aligned}
$$

so the inclusion in the conclusion of the above theorem is not verified.

To conclude this section, we see that only few systems verify the conditions for continuity, that is there exist large classes of switching systems as in (4), with discontinuities on the boundaries of the sets $\chi_{i}$. It is therefore important to propose a framework that encompasses discontinuous vector fields and possible sliding modes when considering switching systems. 


\section{The discontinuous case: the general framework of Filippov}

The conditions that guarantee that the vector field is continuous at the boundaries are somewhat stringent, hence one infers that the case of interest which occurs frequently in practice, is the discontinuous case. A first approach is to embed the system (2) into the class of so-called Filippov's differential inclusions. This however has some drawbacks:

- When the switching surface is attractive and has codimension larger than 2, the uniqueness of solutions is in general not provable, because Filippov's criterion for uniqueness is limited to codimension 1 sliding surfaces. Multiple "sliding" solutions may exist [3, Example 2.30].

- The numerical computation of the solutions of Filippov's inclusions may not be easy.

- The mathematical formalism of (2) does not lend itself very well to the analysis with general properties of operators like dissipativity, maximal monotonicity. More compact formalisms are often much more suitable for the mathematical analysis and the numerical simulation.

This has motivated a number of mathematicians to work on other, more specific classes of discontinuous systems. They usually form only a narrow subclass of switching systems as in (2), however they are tractable from the numerical point of view, and uniqueness of the solutions can be stated. Before going on let us recall some basic facts about Filippov's inclusions.

\subsection{Filippov's differential inclusions}

When considering discontinuous vector fields, the classical notion of solution for ordinary differential equations is too restrictive. Thus, depending on the specific problem at hand, several concepts of a solution associated with a discontinuous system have been introduced. In this section we recall two basic concepts: Caratheodory solution and Filippov solution. For the beginning, let us suppose that the discontinuous system (2) is well-defined over the whole space, i.e. that $f$ is defined on $\mathbb{R}^{n}$.

Definition 5.1. (Caratheodory solution). Given an initial state $x(0)=x_{0}$, a function $x:[0, \infty) \rightarrow \mathbb{R}^{n}$ is a solution of the discontinuous system (2) in the sense of Caratheodory, if it is absolutely continuous on each compact subinterval of $[0, \infty)$ and satisfies (2) almost everywhere.

It happens that Caratheodory solutions are often not sufficient to assure the global existence (i.e. for all $t \geq t_{0}$ ) of the solutions. A well-known example is given by the scalar system:

$$
\dot{x}(t)=\left\{\begin{array}{c}
1 \text { if } x \leq 0 \\
-1 \text { if } x \geq 0 \\
\frac{1}{2} \text { if } x=0
\end{array}\right.
$$

Let $x(0)=1$ and let $f$ be given by the right-hand side in (8). Then $x$ decreases until it attains (after a finite time) the value $x(1)=0$. One is tempted to state that $x$ should then stay at the fixed point $x^{*}=0$ because the trajectory cannot leave the origin (if it 
increases then it has to decrease immediately in view of the vector field in the negative axis). The problem is that $x(t)=0$ for all $t$ (and $\dot{x}(t)=0$ as well) is not a solution of (8) since $0 \notin f(0)$. The Caratheodory solution exists only on the time interval $[0,1)$. Let now $f(0)=0$ in (8). A solution starting at $x(0)=1$ decreases until it attains $x(t)=0$ at $t=1$. The origin $x^{*}=0$ is a solution of the system since $f(0)=0$. Thus the solution stays at $x=0$ for all $t \geq 1$ : the Caratheodory solution exists globally. This has motivated Filippov (see his book [40]) to introduce the set-valued map

$$
F(y)=\bigcap_{\epsilon>0} \bigcap_{\mu(N)=0} \overline{c o} f((y+\epsilon B) \backslash N)
$$

where the intersection is taken over all sets $N \subset \mathbb{R}^{n}$ of (Lebesgue) measure zero and over all $\epsilon>0$. The Filippov inclusion is usually called a convexification of the problem. With system (2), we associate the differential inclusion (known as Filippov's inclusion),

$$
\dot{x}(t) \in F(x(t)) .
$$

For (8), the set is simply $F(x)=\left\{\begin{array}{cl}1 & \text { if } x \leq 0 \\ -1 & \text { if } x \geq 0 \\ {[-1,1]} & \text { if } x=0\end{array}\right.$. This corresponds to fillingin the vertical segment in the graph of the right-hand side. The obtained multifunction is sometimes called the relay function. One notices from (10) that Filippov's construction neglects the value of the discontinuous vector field on the switching surface. This may change the system's dynamics. Indeed let $f(0)=0, f(x)=1$ if $x \neq 0$. A solution starting at $x(0)=-1$ increases until it attains $x(t)=0$ at $t=1$. The trivial solution $x=0$ is a solution of the system since $f(0)=0$. Thus the solution stays at $x^{*}=0$ for all $t \geq 1$ : the Caratheodory solution exists globally. However the Filippov inclusion for this system is $F(x)=1$ for all $x$ and it has no fixed point. With $x(0)=-1$ one gets $x(t)=t-1$ for all $t \geq 0$. Embedding (2) into Filippov's inclusions may involve some modeling assumption.

Definition 5.2. (Filippov solution) An absolutely continuous function $x: \mathbb{R}^{+} \rightarrow \mathbb{R}^{n}$ is said to be a (generalized) Filippov solution of the discontinuous system (2), if it is a solution of differential inclusion (10) for almost all $t \geq 0$, satisfying the initial condition $x(0)=x_{0} \in \mathbb{R}^{n}$.

Since $F$ in (9) is upper semicontinuous (see Proposition 2.2 in [40]), with closed convex values, for any initial condition $x_{0}$, the differential system (2) always has a solution in the sense of Filippov. See e.g. [37] for a clear exposition of the various behaviours that may occur in a Filippov differential inclusion: attractive, repulsive surfaces, and transversal intersections (called hereafter crossing surfaces). Several criteria for the uniqueness of solutions have been stated in [40, Section 10]. They rely on two main arguments: either some kind of monotonicity of the multifunction $F(x)$, known as the one-sided Lipschitz continuity [2]; or, in the case of a codimension 1 switching surface $\Sigma$, the fact that $\Sigma$ is either an attractive surface, or a crossing surface.

Example 5.3. The scalar system with $F(x)=-1$ if $x<0, F(x)=1$ if $x>0$, $F(0)=[-1,1]$ has three solutions at each time $t \geq 0$ such that $x(0)=0: x(t)=0$, or $x(t)=t$, or $x(t)=-t$. This shows that Filippov's differential inclusions may be far from enjoying the uniqueness of solutions properties. This can be generalized to so-called repulsive switching surfaces which yield spontaneous jumps [37]. 
The concept of a Filippov's solution is important in order to analyze sliding phenomena. Nevertheless, we point out that the solution in the sense of Filippov to differential systems with discontinuous right-hand side is continuous in time. This implies that if a solution attains an attractive surface in finite time, it is forced to remain there and slide on it. Systems with a discontinuous solution, i.e. jumps in the state at a certain time instance, are not described by the theory of Filippov.

\subsection{Calculation of a selection in the codimension $\geq 2$ case}

As alluded to above, the drawback of the general Filippov framework is that the differential inclusion may not enjoy the uniqueness of solutions property on attractive surfaces of codimension $\geq 2$. This is due to an ambiguity in defining the sliding vector field. As we shall see later with relay and complementarity systems, there are classes of switching systems for which the ambiguity does not exist, whatever the switching surface codimension. Within the general switching systems class (2) other solutions exist to suppress the ambiguity, see [37] for a review and references.

\subsection{Conditions that guarantee no sliding modes}

Sliding trajectories play a quite important role in switching systems, because they decrease the system's dimension. Moreover they destroy the time-reversibility of the system since several different trajectories may attain in finite-time the same attractive surface. They are created by attractive surfaces which are attained in finite time, which is a peculiar feature of discontinuous switching systems [59]. Besides the possible continuity at the boundaries, one may study the conditions under which the trajectories cross the boundaries, and do not stay on them. Roughly speaking, a trajectory that attains a boundary surface may do it with various degrees of tangency, and leave it similarly to enter another cell. More details will be given in Section 13. Consider the simplest case $n=2, f_{1}(x)=A_{1} x, f_{2}(x)=A_{2} x, \chi_{1}=\left\{x \in \mathbb{R}^{2}: H x+h>0\right\}$, $\chi_{2}=\left\{x \in \mathbb{R}^{2}: H x+h<0\right\}, H \in \mathcal{M}_{1,2}, h \in \mathbb{R}:$ the plane is divided in two cells separated by a line. Then the conditions for crossing at a point $x(t)$ satisfying $H x(t)+h=0 \Leftrightarrow x(t)=-H^{+} h+v(t)$ with $v(t) \in \operatorname{Ker}(H)$ and $H^{+}=H^{T}\left(H H^{T}\right)^{-1}$, are as follows:

$$
\left\{\begin{array}{l}
\left(H^{T} A_{1} x(t), H^{T} A_{1}^{2} x(t), \ldots, H^{T} A_{1}^{i} x(t), \ldots .\right) \succ 0 \text { and } \\
\left(H^{T} A_{2} x(t), H^{T} A_{2}^{2} x(t), \ldots, H^{T} A_{2}^{i} x(t), \ldots .\right) \succ 0 \\
\text { or } \\
\left(H^{T} A_{1} x(t), H^{T} A_{1}^{2} x(t), \ldots, H^{T} A_{1}^{i} x(t), \ldots .\right) \prec 0 \text { and } \\
\left(H^{T} A_{2} x(t), H^{T} A_{2}^{2} x(t), \ldots, H^{T} A_{2}^{i} x(t), \ldots .\right) \prec 0 .
\end{array}\right.
$$

Remark 5.4. The idea of looking at higher order derivatives is ubiquitous when dealing with systems that attain some boundary surface: sliding mode systems [37], systems with complementarity conditions and unilateral constraints [28], systems with Coulomb's friction [42], etc.

\section{The class of Affine Variational Systems}

It is possible to start from other, quite different definitions of nonsmooth dynamical systems than the one in (2). One of these consists in coupling a smooth system to a variational inequality, which we name affine variational systems (AVS). It will be seen 
that some of these AVS can be recast into the general class of discontinuous systems (2).

Let $K$ be a nonempty subset of the Euclidean space $\mathbb{R}^{l}$. An AVS is described by a multi-input multi-output (MIMO) system of the form

$$
\left\{\begin{array}{l}
\dot{x}(t)=g(x(t))+B \lambda(t) \\
y(t)=C x(t)+c+D \lambda(t) \\
x(0)=x_{0} \in \mathbb{R}^{n},
\end{array}\right.
$$

constrained by a variational inequality

$$
(s-\lambda(t))^{T} y(t) \geq 0, \quad \forall s \in K,
$$

where $g():. \mathbb{R}^{n} \rightarrow \mathbb{R}^{n}, B \in \mathcal{M}_{n, l}(\mathbb{R}), C \in \mathcal{M}_{l, n}(\mathbb{R}), D \in \mathcal{M}_{l, l}(\mathbb{R})$ and $c \in \mathbb{R}^{l}$ is a constant. The class of AVS in (12)-(13) represents in fact a large class of nonsmooth dynamical systems, that is impossible to analyze without being more specific on its ingredients like $D$ and the set $K$. They may in turn be seen as a subclass of differential variational inequalities (DVI) [61]. The two main well-posedness results for DVI in [61] are Theorem 6.1 (existence of weak Caratheodory solutions) and Proposition 5.1 (existence and uniqueness of $\mathcal{C}^{1}$ solutions). In the following we present results that assure both existence and uniqueness, and we focus on two particular classes of AVS only. Let us notice that if $K$ is a convex closed set, the variational inequality (13) is equivalent to the "normal cone" inclusion

$$
-y(t) \in N_{K}(\lambda(t)),
$$

that comes simply from the definition of the normal cone to a convex set [2]. This inclusion is in turn equivalent to

$$
\lambda(t) \in-N_{K^{*}}(y(t)),
$$

and to the cone complementarity relation

$$
K^{*} \ni y(t) \perp \lambda(t) \in K,
$$

where the script $\perp$ means "perpendicular" and $K^{*}$ is the positive dual cone of $K$, defined by

$$
K^{*}=\left\{v \in \mathbb{R}^{l}: k^{T} v \geq 0 \forall k \in K\right\} .
$$

The AVS in (12)-(13) may belong to the class of switching systems (2) as the following examples show.

Example 6.1. Let us consider $K=\mathbb{R}^{+}, n=1, d>0$; one has:

$$
\left\{\begin{array}{l}
\dot{x}(t)=a x(t)+b \lambda(t) \\
y(t)=C x(t)+c+d \lambda(t) \\
0 \leq \lambda(t) \perp y(t) \geq 0
\end{array}\right.
$$

For such an AVS the second and third line define a Linear Complementarity Problem with unknown $\lambda(t): 0 \leq \lambda(t) \perp C x(t)+c+d \lambda(t) \geq 0$. It is easy to find by inspection that the following holds: if $C x(t)+c \geq 0$ then $\lambda(t)=0$, if $C x(t)+c \leq 0$ then $\lambda(t)=\frac{1}{d}(-C x(t)-c)$. Therefore (17) is a switching system of the form:

$$
\dot{x}(t)= \begin{cases}a x(t) & \text { if } C x(t)+c \geq 0 \\ \left(a-\frac{b}{d} C\right) x(t)-\frac{b c}{d} & \text { if } C x(t)+c \leq 0\end{cases}
$$

RR $n^{\circ} 7760$ 
This vector field is continuous on the switching surface and it can be checked that the conditions exposed in Section 4 hold. As we shall see next, the continuity may be inferred from another argument that involves either the properties of the solutions of LCPS (complementarity theory), or projection onto a convex set (convex analysis).

Example 6.2. Let us consider $K=[\alpha, \beta], \alpha<\beta, n=1$ and

$$
\left\{\begin{array}{l}
\dot{x}(t)=a x(t)+b \lambda(t) \\
y(t)=C x(t)+c \\
-y(t) \in N_{K}(\lambda(t))
\end{array}\right.
$$

In order to eliminate the unknown $\lambda(t)$ (that may be considered as a Lagrange multiplier) one needs to invert the inclusion. This may be done using convex analysis. The conjugate function of the indicator function $\psi_{K}$ of the set $K$ is the convex function $\psi_{K}^{*}(z)=\sup _{x \in[\alpha, \beta]} z x$ so that $\psi_{[\alpha, \beta]}^{*}(z)=\beta z$ if $z \geq 0$ and $\psi_{[\alpha, \beta]}^{*}(z)=\alpha z$ if $z \leq 0$. The subdifferential of $\psi_{[\alpha, \beta]}^{*}$ in the sense of convex analysis is the multivalued function $\partial \psi_{[\alpha, \beta]}^{*}(z)=\beta$ if $z>0, \partial \psi_{[\alpha, \beta]}^{*}(z)=\alpha$ if $z<0$, and $\partial \psi_{[\alpha, \beta]}^{*}(0)=[\alpha, \beta]$. The characteristic of $\partial \psi_{[\alpha, \beta]}^{*}$ is as in Figure $2(a)$. From convex analysis it follows that the inclusion in (19) is equivalent to $\lambda(t) \in \partial \psi_{[\alpha, \beta]}^{*}(-y(t))$. One may verify that this corresponds to an inversion of the normal cone inclusion (see for instance Figure 1.9 in [2] or Figure 2.11 in [3] for a graphical illustration of such a process). Therefore (19) is equivalently rewritten as:

$$
\dot{x}(t) \in a x(t)+b \partial \psi_{[\alpha, \beta]}^{*}(-C x(t)-c)
$$

whose vector field is discontinuous at $C x+c=0$. Indeed we have:

$$
\dot{x}(t) \in \begin{cases}\{a x(t)+\beta b\} & \text { if } C x(t)+c<0 \\ \{a x(t)+\alpha b\} & \text { if } C x(t)+c>0 \\ a x(t)+b[\alpha, \beta] & \text { if } C x(t)+c=0\end{cases}
$$

The formulation of the AVS provides automatically a way to define what happens on the switching surface: the right-hand side of (21) is multivalued at $C x+c=0$ where the graph of the discontinuous vector field has been "filled-in". One could have started with the definition of the discontinuous system in (21), and then compute its associated Filippov's set to obtain the same result at $C x+c=0$. Notice that the multifunction $\partial \psi_{[\alpha, \beta]}^{*}$ is maximal monotone. One sees that the system in (18) belongs to the class of systems in (2), where the dynamics on the switching surface boundary has been accurately defined.

Example 6.3. Let us now consider the same system as in (19), with $D=d>0$ :

$$
\left\{\begin{array}{l}
\dot{x}(t)=a x(t)+b \lambda(t) \\
y(t)=C x(t)+c+d \lambda(t) \\
-y(t) \in N_{K}(\lambda(t))
\end{array}\right.
$$

The inclusion is now $C x(t)+c+d \lambda(t) \in-N_{[\alpha, \beta]}(\lambda(t))$. In order to solve it (this is a generalized equation with unknown $\lambda(t)$ ), we may rely on a basic relation of convex analysis as follows. Let $x \in \mathbb{R}^{n}, y \in \mathbb{R}^{n}, D=D^{T}>0, K \subseteq \mathbb{R}^{n}$ non empty, closed and convex. Then:

$$
\begin{aligned}
-x+y \in D^{-1} N_{K}(x) & \Leftrightarrow x=\operatorname{proj}_{D}(K ; y) \\
& \Leftrightarrow x=\operatorname{argmin}_{z \in K} \frac{1}{2}(z-y)^{T} D(z-y)
\end{aligned}
$$

RR $n^{\circ} 7760$ 
where $\operatorname{proj}_{D}(K ; y)$ means the projection of $y$ on $K$ in the metric defined by $D$. Using (23) one sees that it is possible to rewrite (22) as:

$$
\dot{x}(t)=a x(t)+b \operatorname{proj}\left([\alpha, \beta] ;-\frac{C x(t)+c}{d}\right) .
$$

The projection operator being a Lipschitz continuous function, it is obvious that the right-hand side of (24) is continuous. So once again the system (22) belongs to the class of switching systems (2) but with a continuous vector field.

Example 6.4. Let us reconsider Example 6.1 with $d=0$ :

$$
\left\{\begin{array}{l}
\dot{x}(t)=a x(t)+b \lambda(t) \\
y(t)=C x(t)+c \\
0 \leq \lambda(t) \perp y(t) \geq 0 .
\end{array}\right.
$$

The inclusion now reads as $C x(t)+c \in-N_{K}(\lambda(t))$ that is equivalent (since $\left.K=\mathbb{R}^{+}\right)$to $\lambda(t) \in-N_{K}(C x(t)+c)$. The AVS in (25) is therefore the differential inclusion:

$$
-\dot{x}(t)+a x(t) \in N_{K}(C x(t)+c) .
$$

There is a strong discrepancy between the case $d>0$ and the case $d=0$. Consider that $C x(t)+c \in K$. Then at time t one gets $-\dot{x}(t)+a x(t)=0$, because $N_{K}(C x(t)+$ $c)=\{0\}$, the null vector of $\mathbb{R}^{n}$. Suppose now that $C x(t)+c \in \partial K$, i.e. the system evolves on the boundary of $K$. This is possible if for instance $a<0$ and $C=1, c=0$ (we may also add a time-varying term (a sort of control action) to complicate a little the dynamics). Then $N_{K}(C x(t)+c)$ is the set $\{z \in \mathbb{R}: z \leq 0\}$, since $N_{K}(0)=\mathbb{R}^{-}$. The case $C x(t)+c \notin K$ is excluded, as one may just define that the normal cone is the empty set. The complementarity system (25) is thus equivalent to:

$$
-\dot{x}(t)+a x(t) \in\left\{\begin{array}{l}
\{0\} \text { if } C x(t)+c>0 \\
\mathbb{R}^{-} \text {if } C x(t)+c=0
\end{array} .\right.
$$

The system switches between an ordinary differential equation (here linear invariant), and a differential inclusion into a normal cone.

What is to be learned from these four simple examples? Essentially that AVS as in (12)-(13) contains an important subclass that belongs to switching systems as in (2). And that this depends heavily on $D$ and $K$. Let us come back to (12) coupled to (14), that involves the generalized equation $-C x-c-D \lambda \in N_{K}(\lambda)$. Following [17] we may rewrite equivalently this inclusion as:

$$
\lambda \in\left(D \cdot+N_{K}\right)^{-1}(-C x-c)
$$

and the AVS as the differential inclusion:

$$
\dot{x}(t) \in g(x(t))+B\left(D \cdot+N_{K}\right)^{-1}(-C x(t)-c) .
$$

The above examples show that depending on $D$ and $K$, the operator in (28) may be single valued and continuous, or multivalued. Its domain may not be the whole of $\mathbb{R}^{n}$. In the above examples we have not specified the initial condition $x(0)=x_{0}$. Actually $x_{0} \in \operatorname{Dom}(\varphi), \varphi: x \mapsto\left(D \cdot+N_{K}\right)^{-1}(-C x-c)$. In Examples 6.1, 6.2, and 6.3, the domain is $\mathbb{R}$. In Example 6.4 the domain is restricted to those $x_{0}$ such that $C x_{0}+c \in \mathbb{R}^{+}$. 
There is another thing to be learned from these four examples: some AVS keep their dimension (Examples 6.1 and 6.3) while others may live on low-dimensional subspaces (Examples 6.2 and 6.4). The case of Example 6.2 with $a=0, \alpha<0, \beta<0, b>0$, is common in switching systems (2) where attractive sliding surfaces which are attained in finite time exist. We say that a system lives on a low-dimensional subspace if there exists a time interval $\left[t_{0}, t_{1}\right], t_{1}>t_{0}$, such that $h(x(t))=0$ for all $t \in\left[t_{0}, t_{1}\right]$, where $h: \mathbb{R}^{n} \rightarrow \mathbb{R}$ is a $\mathcal{C}^{1}$ function. In other words, the system is subject to state equality constraints on positive time intervals. In the case of Example 6.4, the state is subject to an inequality $C x+c \geq 0$ defining an admissible domain for $x$, and may evolve on the boundary of this domain depending on $a, C$ and $c$. We shall come back later on the differential inclusions as in (26), that do not fit with (2).

It is quite interesting to compare the conditions in Example 6.3 that guarantee that the right-hand-side is continuous, and those of Theorems 4.1 and 4.4. The positive definiteness of $D$ assures directly the continuity at the boundaries of the cells, without having to check the conditions one by one. Other "global" conditions assuring continuity (so that the right-hand-side is single-valued) will be given later in Sections 8 and 10.

We have not yet presented any well-posedness results. In the next two sections we focus on two specific classes of AVS, that correspond to $K$ a closed rectangle (relay systems) and $K$ a polyhedral cone (complementarity systems), and provide some existence and uniqueness of solutions results that have been proposed in the literature.

\section{Relay systems}

Relay systems are widely used in Systems and Control, because they allow one to design robust discontinuous feedback controllers [60]. They may also model some mechanical systems with one-dimensional Coulomb friction [2], or electrical circuits with ideal Zener diodes [3]. Let $K$ be a closed rectangle, given by $K=\left\{\lambda \in \mathbb{R}^{l} ; \alpha_{i} \leq\right.$ $\left.\lambda_{i} \leq \beta_{i}, i \in \overline{1, l}\right\}$, with $\alpha_{i}<\beta_{i}, \alpha_{i}, \beta_{i} \in \mathbb{R}$. Let us first use some convex analysis to rewrite the AVS under a relay system form. Similar calculations have been made in Example 6.2 to invert the multivalued part of the system. Let $f_{i}$ be proper convex such that $f_{i}\left(z_{i}\right)=\alpha_{i} z_{i}$ if $z_{i} \leq 0$, and $f_{i}\left(z_{i}\right)=\beta_{i} z_{i}$ if $z_{i} \geq 0$. Let $f(z)=$ $f_{1}\left(z_{1}\right)+\ldots+f_{l}\left(z_{l}\right)$. Then the subdifferential of $f$ is $\partial f(z)=\partial f_{1}\left(z_{1}\right)+\ldots+\partial f_{l}\left(z_{l}\right)$, and $\partial f_{i}\left(z_{i}\right)=\alpha_{i}$ if $z_{i}<0, \partial f_{i}\left(z_{i}\right)=\beta_{i}$ if $z_{i}>0, \partial f_{i}(0)=\left[\alpha_{i}, \beta_{i}\right]$. If $\alpha_{i}=-1$ and $\beta_{i}=1$, then $\partial f_{i}\left(z_{i}\right)$ is the multivalued sign function, or relay multifunction. Then we may denote $\partial f(z)=\operatorname{Sgn}(z)$ with $\operatorname{Sgn}(z)=\left(\operatorname{Sgn}\left(z_{1}\right), \ldots, \operatorname{Sgn}\left(z_{l}\right)\right)^{T}$. By extension and some abuse of notation we shall denote $\partial f_{i}\left(z_{i}\right)$ as $\operatorname{Sgn}\left(z_{i}\right)$ for any $\alpha_{i}$ and $\beta_{i}$. Now we have from convex analysis that $\xi \in \partial f(z) \Leftrightarrow z \in \partial f^{*}(\xi)$, where $f^{*}$ is the conjugate of $f$. Here $f^{*}(\xi)=\psi_{\left[\alpha_{1}, \beta_{1}\right]}\left(\xi_{1}\right)+\ldots+\psi_{\left[\alpha_{1}, \beta_{1}\right]}\left(\xi_{l}\right)$, so that $\partial f^{*}(\xi)=\left(N_{\left[\alpha_{1}, \beta_{1}\right]}\left(\xi_{1}\right), \ldots, N_{\left[\alpha_{l}, \beta_{l}\right]}\left(\xi_{l}\right)\right)^{T}=N_{K}(\xi)$. From (14) we deduce that $-y(t) \in N_{K}(\lambda(t))$ is equivalent to $-y(t) \in \partial f^{*}(\lambda(t))$ so $\lambda(t) \in \partial f(-y(t))$ and $\lambda_{i}(t) \in \operatorname{Sgn}\left(-y_{i}(t)\right)$, where $\lambda_{i}(t)$ and $y_{i}(t)$ stand for the $i$-th component of $\lambda(t)$ and of $y(t)$, respectively. The AVS in (12)-(13) is therefore equivalent to the so-called relay system:

$$
\left\{\begin{aligned}
\dot{x}(t) & =g(x(t))+B \lambda(t) \\
y(t) & =C x(t)+c+D \lambda(t) \\
\lambda_{i}(t) & \in \operatorname{Sgn}\left(-y_{i}(t)\right), \quad i \in \overline{1, l} \\
x(0) & =x_{0} \in \mathbb{R}^{n} .
\end{aligned}\right.
$$



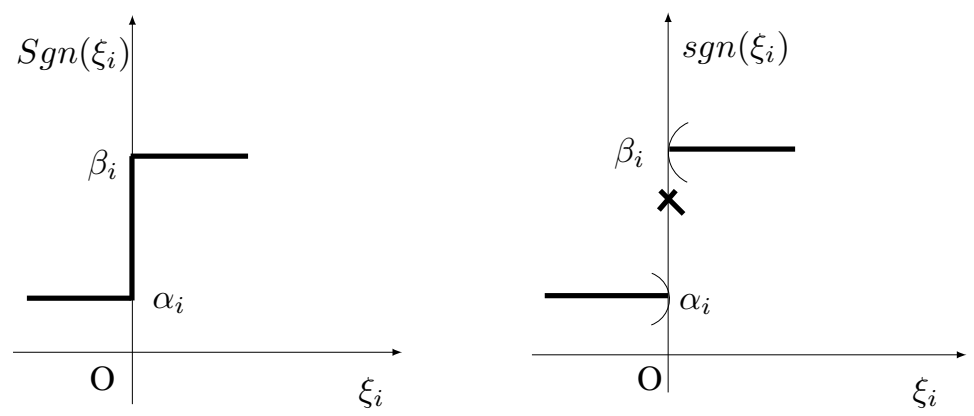

Figure 2: (a) The general relay characteristics. (b) The general relay single-valued map.

For $i \in \overline{1, l}$, each pair $\left(\lambda_{i}, y_{i}\right)$ satisfies a general relay characteristic, as depicted in Figure 2 (a). Notice that if $\alpha_{i}=-\beta_{i}, \beta_{i}>0$, then $\operatorname{Sgn}\left(-y_{i}(t)\right)=-\operatorname{Sgn}\left(y_{i}(t)\right)$. Moreover, the graph of the sign multifunction in Figure 2 (a) is maximal, while the one in Figure 2 (b) is not maximal, but both are monotone.

From the above four examples, a question emerges naturally: when is the general system (30) a switching system of the form (2) and how can we describe the cells in this case? To address this question, we reconsider the relay system (30) as a special class of AVS and use the constructive theory of Affine Variational Problems. First we rewrite inequality (13) as

$$
(s-\lambda(t))^{T}(C x(t)+c+D \lambda(t)) \geq 0, \quad \forall s \in K .
$$

Let us introduce some notions we use hereafter.

Definition 7.1. [38] Let $f: \mathbb{R}^{l} \rightarrow \mathbb{R}^{l}$ be a continuous function such that there exists a finite family of affine functions $\left\{f^{1}, \ldots, f^{k}\right\}$ that maps $\mathbb{R}^{m}$ into itself and for every $x \in \mathbb{R}^{l}$ there is an $i \in \overline{1, k}$ such that $f(x)=f^{i}(x)$. Then, $f$ is said to be piecewise affine (PWA).If, in addition, $\operatorname{det}\left(J f^{i}\right)$ has the same nonzero sign for all $i \in \overline{1, k}$, then the PWA function $f$ is said to be coherently oriented.

The next theorem is a particular case of the results in [61] and is recalled in detail here, because it contains the conditions under which the generalized equation in (31), written as a variational inequation, possesses a unique solution whatever the data $C x(t)+c$.

Theorem 7.2. If $g: \mathbb{R}^{n} \rightarrow \mathbb{R}^{n}$ is Lipschitz continuous and $D \in \mathcal{M}_{l, l}(\mathbb{R})$ is a P-matrix, then the relay system (30) has a unique $\mathcal{C}^{1}$ solution.

Proof. Since $D$ is a $P$-matrix, according to Example 4.2 .9 in [38], the normal map associated to the pair $(K, D)$, given by

$$
M_{K}^{n o r}: \mathbb{R}^{l} \rightarrow \mathbb{R}^{l}, M_{K}^{n o r}(\lambda)=D \cdot \Pi_{K}(\lambda)+\lambda-\Pi_{K}(\lambda),
$$

where $\Pi_{K}(\lambda)=\operatorname{proj}(K ; \lambda)$ (the projection of $\lambda$ on $K$ with respect to the Euclidian metric) is coherently oriented. Then, by Theorem 4.3.2 in [38], the affine variational inequality (31) has a unique PWA solution:

$$
\lambda(C x(t)+c)=\Pi_{K}\left(\left(M_{K}^{n o r}\right)^{-1}(-C x(t)-c)\right)
$$


and thus, Lipschitz continuous, as a function of $C x(t)+c$. Putting $v(x)=\lambda(C x+c)$ we get that also $x \mapsto v(x)$ is Lipschitz continuous. The relay system (30) becomes

$$
\left\{\begin{aligned}
\dot{x}(t) & =g(x(t))+B \Pi_{K}\left(\left(M_{K}^{n o r}\right)^{-1}(-C x(t)-c)\right) \\
y(t) & =C x(t)+c+D \Pi_{K}\left(\left(M_{K}^{\text {nor }}\right)^{-1}(-C x(t)-c)\right) \\
x(0) & =x_{0} \in \mathbb{R}^{n} .
\end{aligned}\right.
$$

The right-hand side in the first equation of (32) is a Lipschitz continuous function, hence we obtain that the solution of (32) is of class $\mathcal{C}^{1}$ in time. Moreover, since the solution of (31) is PWA, from Proposition 4.2.1 in [38] we get that there exists a polyhedral subdivision $\left\{\chi_{i}\right\}_{i \in \overline{1, m}}$ of $\mathbb{R}^{n}$ and a finite family of affine functions $\left\{f_{i}\right\}_{i \in \overline{1, m}}$ such that the right-hand side in the state equation in (30) coincides with $f_{i}$ on each cell $\chi_{i}$. Finally, we recall that each polyhedron $\chi_{i}$ could be described by a finite number of affine inequalities in $\mathbb{R}^{n}$ like in Section 3.1.

Remark 7.3. The above theorem says that the Lipschitz continuity of $g$ together with the P-matrix property of D are sufficiently strong to guarantee the existence and even the uniqueness of classical solutions.

Let us come back on the generalized equation (31) that we rewrite equivalently as the inclusion:

$$
C x+c+D \lambda \in-N_{K}(\lambda) .
$$

In the scalar case one has $K=[\alpha, \beta]$, and let us denote $D$ as $d$. The uniqueness of solutions depends greatly on the sign of $d$ as depicted in Figure 3. The solution $\lambda$ corresponds to the intersections between the graph of the maximal monotone operator $\lambda \mapsto N_{K}(\lambda)$ and the affine maps $\lambda \mapsto-C x-c-d \lambda$. If $d>0$ there is always a unique solution; if $d<0$ there may be multiple solutions. This is a particular case of a more general result on existence and uniqueness of solutions of $0 \in F(\lambda)$, that is guaranteed if $F$ is a maximal monotone operator [38]. When $D=D^{T}>0$ the solution is calculated as in (23).

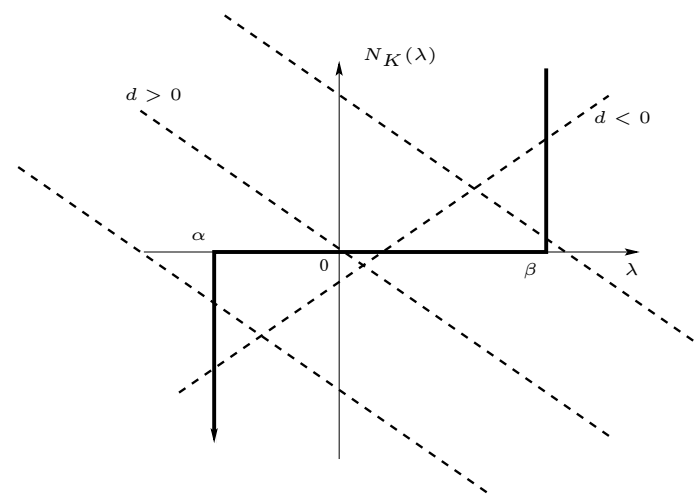

Figure 3: Uniqueness and non uniqueness of solutions for (33). 


\subsection{Linear relay systems}

In the particular case when $g$ is a linear function given by $g(x)=A x$ with $A \in$ $\mathcal{M}_{n, n}(\mathbb{R})$ and $c=0$, the well-posedness of the above system was studied in [55], [54] and [65].

Definition 7.4. A triple $(\lambda, y, x):[0, \infty) \rightarrow \mathbb{R}^{l} \times \mathbb{R}^{l} \times \mathbb{R}^{n}$ is called a forward solution to the relay system (30), ift $\mapsto x(t)$ is continuous on $[0, \infty)$ and there exists a countable number of switching times $0=t_{0}<t_{1}<\ldots<t_{j}<\ldots$ such that for every interval $\left[t_{j}, t_{j+1}\right)$ the triple $(\lambda, y, x)$ verifies the following conditions:

i) For any $i \in \overline{1, l}$ and $t \in\left[t_{j}, t_{j+1}\right), \lambda_{i}(t)$ and $y_{i}(t)$ correspond to one and only one of the following three branches:

$$
\begin{gathered}
{\left[y_{i}(t)>0 \text { and } \lambda_{i}(t)=\alpha_{i}\right] \text { or }\left[y_{i}(t)<0 \text { and } \lambda_{i}(t)=\beta_{i}\right] \text { or }} \\
{\left[y_{i}(t)=0 \text { and } \lambda_{i}(t) \in\left[\alpha_{i}, \beta_{i}\right]\right] .}
\end{gathered}
$$

ii) $(\lambda, y, x)$ is analytic (on $\left.\left[t_{j}, t_{j+1}\right)\right)$.

iii) $(\lambda, y, x)$ verifies (30) with initial condition $x\left(t_{j}\right)=\lim _{t \nearrow t_{j}} x(t)$. For $j=0$ the initial condition is given in (30) i.e., $x\left(t_{0}\right)=x_{0}$.

We notice that the above definition excludes the existence of right-accumulations of switches (a kind of Zeno behaviour) but allows for left-accumulations. In short, a forward solution is continuous and analytic between the switching instants, that may accumulate on the left. The conditions under which the system (30) admits a unique forward solution are contained in the following theorem.

Theorem 7.5. [54] Let $g(x)=A x, A \in \mathcal{M}_{n, n}(\mathbb{R})$ and $c=0$. Suppose that there exists $s_{0} \geq 0$ such that $G(s)=C\left(s I_{n}-A\right)^{-1} B+D$ is an invertible $P_{0}$-matrix for $s \geq s_{0}$. Then, for any initial condition $x_{0}=x(0)$, the relay system (30) admits a unique forward solution $(\lambda, y, x), t \geq 0$ in the sense of Definition 7.4.

Since the works in $[54,55,65]$ use the notion of a transfer matrix and the linear complementarity theory as basic analysis tools, they are restricted to the "all linear invariant" case, i.e. the vector fields $f_{i}$ have to be linear invariant and the cells $\chi_{i}$ have to be constant polyhedra. The strength of the results lies in the fact that they allow for $P_{0}$ transfer matrices and non zero $D$ matrices. Linear relay systems satisfying the conditions of Theorem 7.5 may exhibit sliding modes or accumulations of switches as the following examples show. However repulsive surfaces as in example 5.3 are not admitted, since they yield non unique analytic solutions.

Example 7.6. Let $n=2, A=0, C=I_{2}, B=\left(\begin{array}{ll}1 & 0 \\ 6 & 1\end{array}\right)$. The transfer matrix is $\frac{1}{s} B$ and $B$ is a $P$-matrix. Then $\dot{x}(t)=B \lambda(t), \lambda_{1}(t) \in-\operatorname{Sgn}\left(x_{1}(t)\right), \lambda_{2}(t) \in-\operatorname{Sgn}\left(x_{2}(t)\right)$ and $[\lambda(t)]^{T}=\left(\lambda_{1}(t), \lambda_{2}(t)\right)$. The four vector fields are easily computable as $(-1-$ $7)^{T},\left(\begin{array}{ll}1 & 5\end{array}\right)^{T},\left(\begin{array}{ll}1 & 7\end{array}\right)^{T},\left(\begin{array}{ll}-1 & -5\end{array}\right)^{T}$ in the first, second, third and fourth quadrants respectively (hence the system belongs to the class in (2)). All trajectories starting outside the axis $x_{1}=0$ attain this line in a finite time and slide on it towards the origin $x=0$. The line $x_{2}=0$ is a crossing surface. See Figure $4(a)$. One sees that in the case of an attractive surface the forward solution matches with the Filippov's solution, because this is the unique possible solution. When attaining the line $x_{1}=0$, the trajectory cannot go back in $x_{1}<0$, it cannot cross to $x_{1}>0$, so it can only slide on $x_{1}=0$. 
Example 7.7. Let $n=2, A=0, C=I_{2}, B=\left(\begin{array}{cc}1 & -2 \\ 2 & 1\end{array}\right)$. The transfer matrix is $\frac{1}{s} B$ and $B$ is a (non symmetric) positive definite matrix, hence a $P$-matrix. The trajectories initialized outside the origin reach it in finite time after an infinity of switches, when the solution crosses the two switching surfaces $x_{1}=0$ and $x_{2}=0$ (see for instance [2]). See Figure 4 (b).

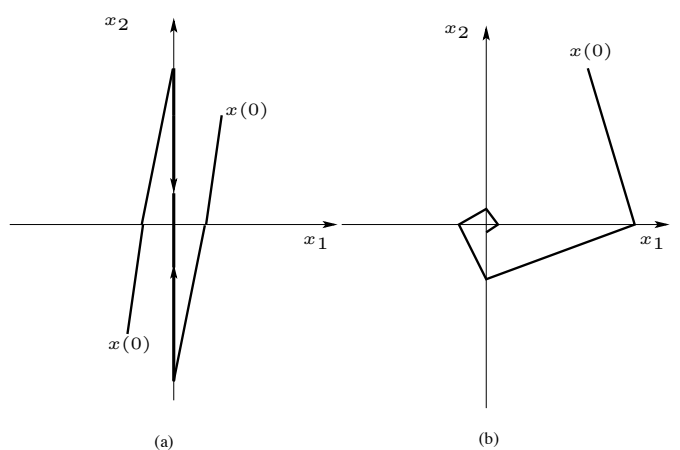

Figure 4: Linear relay systems with (a) sliding motion, and (b) accumulation of switches.

Example 7.6 indicates that the general existence result of Filippov (that states the existence of absolutely continuous solutions) can be refined to the existence of continuous piecewise analytic solutions in some cases. The simplest case that indicates this point is the differential inclusion $\dot{x}(t) \in-\operatorname{Sgn}(x(t))$ that indeed possesses a unique forward solution whatever $x(0)$. This example can also be analyzed with Theorem 7.11 below. Another interesting result is stated in [65] for the single relay case.

Theorem 7.8. [65] Let $y(t)$ and $\lambda(t)$ be scalars, and $D=0$. Then for any initial condition $x(0)$ there exists a unique forward solution if and only if the leading Markov parameter $M_{r}=C A^{r-1} B$ is positive, where $r=\min \left\{i=1,2, \ldots\right.$, such that $C A^{i-1} B \neq$ $0\}$.

The uniqueness of Filippov (hence absolutely continuous) solutions is a different matter, see Section 7.3.

\subsection{Nonlinear relay systems}

In what follows we will study two classes of relay systems for the particular case when $D=0$, so Theorem 7.2 fails to apply. Contrary to the above linear relay systems, they allow for some nonlinearity in their ingredients. When $D=0$, the relay system (30) becomes the differential inclusion

$$
\left\{\begin{array}{l}
\dot{x}(t) \in g(x(t))-B \operatorname{Sgn}(C x(t)+c) \\
y(t)=C x(t)+c \\
x(0)=x_{0} \in \mathbb{R}^{n}
\end{array}\right.
$$

where

$$
\operatorname{Sgn}(C x(t)+c)=\left(\operatorname{Sgn}\left(C_{1} \bullet x(t)+c_{1}\right), \ldots, \operatorname{Sgn}\left(C_{l} \bullet x(t)+c_{l}\right)\right)^{T}
$$


and the graph of $\operatorname{Sgn}\left(y_{i}\right), y_{i}=C_{i \bullet} x+c_{i}$ is depicted in Figure 2 (a). Set $h_{j}(x)=$ $C_{j} \bullet x+c_{j}, j \in \overline{1, l}$ and the relay inclusion is of the form (2).

Remark 7.9. It should be pointed out that the multiplication between the matrix $B$ and the set-valued signum map Sgn is understood in the sense of multiplication of the sets by scalars. More precisely, for $B$ and $\operatorname{Sgn}(C x+c)$ as above, the product $B \operatorname{Sgn}(C x+c)$ is allowed in the following sense:

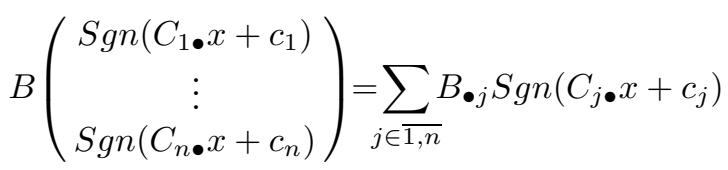

which is different from

$$
\left(\begin{array}{c}
B_{1} \cdot S g n(C x+c) \\
\vdots \\
B_{n} \bullet \operatorname{Sgn}(C x+c)
\end{array}\right)=\sum_{i \in \overline{1, n}} B_{i \bullet} \operatorname{Sgn}(C x+c) e^{i},
$$

where $e^{i}$ stands for the $i$-th canonical unit vector in $\mathbb{R}^{n}$. An illustration of this fact is given in Section 7.4.

Definition 7.10. A solution to the differential relay inclusion (34) is a pair $(x, y)$ of absolutely continuous functions, $x: \mathbb{R} \rightarrow \mathbb{R}^{n}$ and $y: \mathbb{R} \rightarrow \mathbb{R}^{l}$, such that $x$ satisfies the first equation in (34) a.e. on $[0, \infty)$ with initial condition $x(0)=x_{0}$ and the output $y$ satisfies $y(t)=C x(t)+c$ for each $t \geq 0$.

The well-posedness of this class of relay systems was stated in [1], using the maximal monotonicity property of the subdifferential of a certain convex function. The result we give below could be useful when characterizing the Filippov solutions of certain relay systems which will be discussed in Section 7.4.

Theorem 7.11. [1] Suppose that $g$ is Lipschitz continuous and there exists a positive definite matrix $P=P^{T}$ such that $P B=C^{T}$. Then, for an initial condition $x_{0} \in \mathbb{R}^{n}$, the differential inclusion (34) admits a unique Lipschitz solution with an essentially bounded derivative.

Proof. (Sketch of the proof) The main theoretical tool that is used in the proof is the maximal monotonicity of a multivalued operator. The first step consists in performing a suitable state vector change $z=R x$, with $R$ the symmetric positive definite square root of $P$. This has been introduced in [13]. Once this is done, the relay system is put under the following canonical form:

$$
\dot{z}(t) \in g(z(t), t)-F^{T} \operatorname{Sgn}(F z(t)+c)
$$

with $F=C R^{-1}$. The basic convex analysis tool that is used is the chain rule [2, proposition A.3] and then a general result about the existence and uniqueness of solutions for differential inclusions with a maximal monotone multivalued function [9]. We notice from (37) that the vector fields $f_{i}$ take a special form. Indeed suppose that $g(z, t)=0$. We obtain that $\dot{z}(t) \in-\sum_{i=1}^{m} F_{i \bullet}^{T} \operatorname{Sgn}\left(F_{i} \bullet z(t)+c_{i}\right)$. The vector fields $f_{i}$ are obtained from the sum of vector fields orthogonal to the switching surfaces $\Sigma_{i}=F_{i \bullet} z+c_{i}=0$. Suppose further that $F \in \mathbb{R}^{m \times n}$ has full rank $m$, so that $F F^{T}$ is symmetric positive definite. Then the codimension $m$ surface $\cap_{1 \leq i \leq m} \Sigma_{i}$ is attractive and attained in finite 
time. The proof may be led with the function $V(z)=\frac{1}{2}(F z+c)\left(F F^{T}\right)^{-1}(F z+c)$. Along the trajectories of (37) with $g(z, t)=0$ and as long as $z \notin \Sigma_{i}$ for all $1 \leq i \leq m$, one has $\dot{V}(t)=-\sum_{i=1}^{m}\left|F_{i} \bullet z(t)+c_{i}\right|$. Therefore $V$, which is a positive definite function of the variables $\sigma_{i}=F_{i} z+c_{i}$, decreases to zero in finite time.

Suppose that $g(z, t)=A z$ in (37). Then the transfer matrix of Theorem 7.5 is $G(s)=F\left(s I_{n}-A\right)^{-1} F^{T}$. In Theorem 7.11 one does not impose that $G(s)$ should be $P_{0}$, however $D=0$ and $B=F^{T}$. Let us come back to Theorem 7.5. Notice that we may rewrite $G(s)=\frac{1}{s} C\left(I_{n}-\frac{A}{s}\right)^{-1} B+D$ so that for large $s$ one has $G(s) \approx$ $\frac{1}{s} C B+D$. This seems to indicate that when $g(x)=A x$ Theorem 7.5 is more general than Theorem 7.11. However it is noteworthy that Theorems 7.5 and 7.11 do not deal with the same functional spaces of solutions and can therefore hardly be compared from this point of view. Indeed it is known that a relay system can admit unique forward solutions, while it admits several absolutely continuous Filippov solutions, for instance (see Section 7.3). Notice that the system of Example 7.7 does not satisfy the conditions of Theorem 7.11.

Example 7.12. Consider that $B=\left(b_{j i}\right)$ is a diagonal matrix with $b_{i i}>0, \forall i \in \overline{1, n}$, $C=I_{n}$ and $c=0$. Assume also that $g$ is Lipschitz continuous. Then there exists a positive definite matrix $P=B^{-1}$ such that $P B=C^{T}$, and according to Theorem 7.11 , the relay inclusion

$$
\left\{\begin{array}{l}
\dot{x}(t)=g(x(t))-\sum_{i \in \overline{1, n}} b_{i i} S g n\left(x_{i}(t)\right) e^{i} \\
x(0)=x_{0} \in \mathbb{R}^{n}
\end{array}\right.
$$

has a unique Lipschitz solution with an essentially bounded derivative. We will return to this example in remark 7.18, when regarding the above system as the Filippov's regularization of the relay system obtained by replacing the set-valued map signum by its single-valued version.

Let us now investigate the existence of classical solutions for another class of nonlinear relay systems:

$$
\left\{\begin{array}{l}
\dot{x}(t)=g(x(t))+B(x) \operatorname{sgn}(C x(t)+c) \\
x(0)=x_{0} \in \mathbb{R}^{n},
\end{array}\right.
$$

where $g():. \mathbb{R}^{n} \rightarrow \mathbb{R}, B(x)=\left(b_{j i}(x)\right)_{j, i \in \overline{1, n}}$ and $b_{j i}():. \mathbb{R}^{n} \rightarrow \mathbb{R}$ for all $j$ and $i$. Here, $\operatorname{sgn}(C x+c)=\left(\operatorname{sgn}\left(C_{1} \bullet x+c_{1}\right), \ldots, \operatorname{sgn}\left(C_{n} \bullet x+c_{l}\right)\right)^{T}$ and $\operatorname{sgn}\left(y_{i}\right)$ stands for the generalized signum single-valued map, $-\operatorname{sgn}(0) \in\left[\alpha_{i}, \beta_{i}\right]$ whenever $y_{i}=$ $C_{i} \bullet x+c_{i}=0$ (see Figure 2 (b)). Clearly, this system can be recast into (2), by defining $d_{j}(x)=C_{j} \bullet x+c_{j}, j \in \overline{1, n}$.

The next theorem is a consequence of Proposition 1 in [30] that states sufficient conditions for the continuity and smoothness of the right-hand side of a general discontinuous dynamical systems. Let us consider that $C$ is a diagonal matrix with $\operatorname{diag}(C)=\left(C_{11}, \ldots, C_{n n}\right)$ with nonzero diagonal entries.

Proposition 7.13. Consider the system (39). Suppose that $b_{j i}$ and $g$ are smooth functions. If for each $j \in \overline{1, n}, b_{j i}$ verifies

$$
b_{j i}\left(x_{1}, \ldots, x_{i-1},-\frac{c_{i}}{C_{i i}}, x_{i+1}, \ldots, x_{n}\right)=0 \quad \forall i \in \overline{1, n},
$$


then, there exists at least one classical solution to the system (39). If, in addition, $b_{j i}$ verifies

$$
\frac{\partial b_{j i}}{\partial x_{i}}\left(x_{1}, \ldots, x_{i-1},-\frac{c_{i}}{C_{i i}}, x_{i+1}, \ldots, x_{n}\right)=0 \quad \forall i \in \overline{1, n},
$$

then the system (39) admits a unique classical solution on $\mathbb{R}$.

Proof. The functions $x_{i} \mapsto \operatorname{sgn}\left(C_{i} \bullet x_{i}+c_{i}\right)$ are piecewise smooth functions with a single non-smoothness point $\delta_{i}=-\frac{c_{i}}{C_{i i}}$ and $b_{j i}\left(x_{1}, \ldots, x_{i-1}, \delta_{i}, x_{i+1}, \ldots, x_{n}\right)=0$. Hence, due to Proposition 1 in [30], the right-hand side in (39) is a continuous function on $\mathbb{R}^{n}$ and the solutions of the above system are continuously differentiable. Moreover, if $b_{j i}$ verifies relations (40), then the right-hand side in (39) is smooth on $\mathbb{R}^{n}$, hence the system (39) has a unique $\mathcal{C}^{1}$ solution.

To illustrate the applicability of Theorem 7.13 , let us consider the following

Example 7.14. Is is easy to see that if $g$ is a certain smooth function and $b_{j i}(x)=$ $b_{j i}\left(x_{i}+\frac{c_{i}}{C_{i i}}\right)^{2}$ for $j, i \in \overline{1, n}$ then, the conditions from Theorem 7.13 are satisfied, so the nonlinear relay system

$$
\left\{\begin{array}{l}
\dot{x}(t)=g(x(t))+\sum_{i \in \overline{1, n}} B \bullet\left(x_{i}+\frac{c_{i}}{C_{i i}}\right)^{2} \operatorname{sgn}\left(C_{i i} x_{i}(t)+c_{i}\right) \\
x(0)=x_{0} \in \mathbb{R}^{n}
\end{array}\right.
$$

admits a unique classical solution.

Remark 7.15. One can see that the discontinuity of the right-hand side does not necessarily imply the non-existence of classical solutions (for instance if the switching surface is of the crossing type). However, in general, when considering discontinuous systems, classical solutions for IVP (2) might not even exist. This is the case when attractive surfaces, that create sliding trajectories, exist. This is why it is required to consider other notions of solution to a discontinuous system. Another reason for considering generalized notions of solutions is that one would like that a system like $\dot{x}(t)=g(t)-\operatorname{sgn}(x(t))$ possesses global-in-time solutions for a large class of functions $g$. Let us simply state that $|g(t)|<1$ for all $t \geq 0$. Then filling-in the graph of the sign function (Figure $2(b)$ with $\alpha_{i}=-1$ and $\beta_{i}=1$ ) allows one to state the existence and the uniqueness of solutions to $\dot{x}(t) \in g(t)-\operatorname{Sgn}(x(t))$, relying for instance on Theorem 7.11. Keeping the sign function would allow this only for specific $g(t)$.

\subsection{The influence of the relative degree}

The relative degree of a system $\dot{x}(t)=A x(t)+B \lambda(t), y(t)=C x(t)+D \lambda(t), y(t)$ and $\lambda(t)$ scalars, is zero if $D \neq 0$, one if $D=0$ and $C B \neq 0$, and is the smallest integer $r$ such that $C A^{i-1} B=0$ for all $1 \leq i<r$ and $C A^{r-1} B \neq 0$. In the multivariable case one may define a uniform relative degree $r$ in the same way. It is apparent that the results of Theorems 7.5, 7.8 and 7.11 imply some relative degree condition. Indeed as alluded to above the conditions of Theorem 7.5 are satisfied if either $D$ or $C B$ are $P$-matrices, hence full-rank. The condition $P B=C^{T}$ of Theorem $7.11 \mathrm{im}$ -

plies that $C B=B^{T} P B$ that is symmetric positive definite if $B$ has full rank. Thus one may roughly say that the well-posedness results hold with relative degrees 0 or 1. For the case of scalar $y(t)$ and $\lambda(t)$ (a unique feedback relay), the results may be 
refined. Theorem 7.8 states the uniqueness of forward solutions. However Filippov's solutions may not be unique. Roughly speaking, Filippov's solutions are unique when $C B>0$ or $C B=0$ and $C A B>0$ (Theorems 2 and 3 in [65]). The relay system $x^{(3)}(t) \in-\operatorname{Sgn}(x(t))$ has been studied in depth in [65], where the relative degree influence is considered on relay system's well-posedness. Obviously $r=3$. It is shown in [65] that despite $C A^{2} B>0$ there exists an infinity of Filippov's solutions starting at $x(0)=\dot{x}(0)=\ddot{x}(0)=0$. These solutions start with a right-accumulation of switches, something that is not permitted if analyticity is imposed as in forward solutions. It follows from this and Theorem 7.8 that forward and Filippov, absolutely continuous solutions, are quite different one from each other.

Remark 7.16. The loss of uniqueness when the solutions are not analytic, is also met in mechanical systems with frictionless unilateral constraints, where the solutions may "emerge from the emptyness" with a right-accumulation of impacts [7]. Analyticity of the data prevents such spurious phenomena. The discrepancy between the above relay system with relative degree three, and the mechanical systems studied in [7], is that the relay system shows this phenomenon in an intrinsic autonomous way, while in mechanics one needs to add an external force to produce it.

\subsection{Relay inclusions versus Filippov inclusions}

As stipulated in [31], relay inclusions in (34) are Filippov regularizations (convexifications) of the relay systems

$$
\left\{\begin{aligned}
\dot{x}(t) & =g(x(t))-B \operatorname{sgn}(C x(t)+c) \\
y(t) & =C x(t)+c \\
x(0) & =x_{0} \in \mathbb{R}^{n}
\end{aligned}\right.
$$

with the mention that the multiplication $B S g n(C x(t)+c)$ should be meant as in (35). On the opposite, if adopting the multiplication in the sense of (36), such inclusions are larger than the Filippov regularizations of the corresponding relay systems. To illustrate it further, let us consider the following two dimensional example. The dynamics is given by

$$
\left\{\begin{array}{l}
\dot{x}(t)=g(x(t))-\left(\begin{array}{ll}
b_{1} & b_{2} \\
b_{3} & b_{4}
\end{array}\right)\left(\begin{array}{l}
\operatorname{sgnx}_{1}(t) \\
\operatorname{sgn}_{2}(t)
\end{array}\right) \\
y(t)=x(t) \\
x(0)=x_{0} \in \mathbb{R}^{n} .
\end{array}\right.
$$

It is readily seen that the Filippov inclusion associated to the above relay system is

$$
\left\{\begin{array}{l}
\dot{x}(t) \in g(x(t))-\left(\begin{array}{c}
b_{1} \\
b_{3}
\end{array}\right) \operatorname{Sgn}\left(x_{1}(t)\right)-\left(\begin{array}{c}
b_{2} \\
b_{4}
\end{array}\right) \operatorname{Sgn}\left(x_{2}(t)\right) \\
y(t)=x(t) \\
x(0)=x_{0} \in \mathbb{R}^{n},
\end{array}\right.
$$

which is strictly contained in the inclusion derived by using (36),

$$
\left\{\begin{array}{l}
\dot{x}(t) \in g(x(t))-\left(\begin{array}{l}
b_{1} \operatorname{Sgn}\left(x_{1}(t)\right)+b_{2} \operatorname{Sgn}\left(x_{2}(t)\right) \\
b_{3} \operatorname{Sgn}\left(x_{1}(t)\right)+b_{4} \operatorname{Sgn}\left(x_{2}(t)\right)
\end{array}\right) \\
y(t)=x(t) \\
x(0)=x_{0} \in \mathbb{R}^{n},
\end{array}\right.
$$


where $b_{1}, b_{2}$ and $b_{3}, b_{4}$ are multiplied by arbitrary elements in $\operatorname{Sgn}\left(x_{1}(t)\right)$ and $\operatorname{Sgn}\left(x_{2}(t)\right)$, respectively. Simple computations show that for $g \equiv 0, b_{i}=1, i=1 . .4$ and the initial condition $x_{0}=(1,0)^{T}$, system (44) admits a unique constant solution $x(t) \equiv(1,0)^{T}$, while system (45) admits besides this solution, an infinity of solutions parameterized by $\lambda \in[-1,1)$, namely

$$
x(t)=\left\{\begin{array}{ll}
((\lambda-1) t+1,0)^{T} & \text { if } t \in[0,1 /(1-\lambda)] \\
(0,0)^{T} & \text { if } t>1 /(1-\lambda)
\end{array} .\right.
$$

However, when $B$ is a diagonal matrix, the relay inclusion of the form (34) always represents a Filippov regularization, no matter the definition of the product $B S g n(C x(t)+$ $c)$ is employed. In fact, this is still valid for any matrix $B$ verifying $b_{i_{1} j} b_{i_{2} j}=0$ and $b_{i j_{1}} b_{i j_{2}}=0$ for all $i_{1}, i_{2}, j_{1}, j_{2}, i, j \in \overline{1, n}$.

Nevertheless, it appears that only few results are available for the uniqueness of Filippov solutions. Let us return to the class of relay systems described in (39). We notice that by taking $B(x)=\operatorname{diag}\left(b_{11}, \ldots, b_{n n}\right), C=I_{n}$ and $c=0$, we cannot apply Theorem 7.13 to the following system:

$$
\left\{\begin{array}{l}
\dot{x}(t)=g(x(t))+\sum_{i \in \overline{1, n}} B_{\bullet} i \operatorname{sgn}\left(x_{i}(t)\right) \\
x(0)=x_{0} \in \mathbb{R}^{n} .
\end{array}\right.
$$

Thus, the existence and uniqueness of solutions to the discontinuous system (46) will be analyzed through the corresponding system obtained by Filippov regularization, namely

$$
\left\{\begin{array}{l}
\dot{x}(t) \in g(x(t))+\sum_{i \in \overline{1, n}} B_{\bullet} \operatorname{Sgn}\left(x_{i}(t)\right) \\
x(0)=x_{0} \in \mathbb{R}^{n} .
\end{array}\right.
$$

In the next theorem we state the conditions under which the system (46) admits a unique Filippov solution, in the sense of Definition 5.2.

Theorem 7.17. [29] Consider the system (46). The following assertions hold.

i) If $g$ is Lipschitz continuous and satisfies a growth condition, that is there exists $k>0$ such that

$$
\|g(x)\| \leq k(1+\|x\|) \quad \forall x \in \mathbb{R}^{n}
$$

then, there exists a Filippov solution to the system (46).

ii) If, in addition, the matrix $\left(b_{j i}\right)_{j, i \in \overline{1, n}}$ has only non-positive elements, then system (46) admits a unique Filippov solution.

Sketch of the proof. The right-hand side in (46) is u.s.c. with non-empty closed, convex values and for any $i=1 . . n$ the multifunctions $b_{i i} \operatorname{Sgn}\left(x_{i}\right)$ are one-sided Lipschitz continuous. Further, a result of Filippov [40] concerning the uniqueness of solutions for differential equations with one-sided Lipschitz continuous right-hand side is applied.

Remark 7.18. Let us reconsider the relay inclusion in Example 7.12 and suppose, in addition, that the function $g$ satisfies a growth condition. Then, due to Theorem 7.11, the unique Filippov solution of system (38) is Lipschitz continuous, having an essentially bounded derivative. 


\subsection{Another class of nonlinear relay systems}

In Section 7.2. we presented the most common class of nonlinear relay systems, i.e. relay systems which are nonlinear in the single-valued part of the vector field, but which are linear with respect to the multivalued signum function. In contrast to this class, we introduce here another class of relay systems arising in biological models, that describes genetic regulatory networks. The nonlinearity of such systems refers to complex combinations of signum functions that model switch-like interactions between different components of the regulatory network: DNA, RNA, proteins and small molecules. Their general form can be described as follows.

$$
\dot{x}_{i}(t)=-\gamma_{i} x_{i}(t)+\sum_{l \in L_{i}} k_{i l} b_{i l}(x(t)), i=1 . . n,
$$

where $x_{i}$ denotes the cellular concentration of the product gene $i, \gamma_{i}>0$ is the degradation rate of $x_{i}, k_{i l}>0$ is a rate parameter and $L_{i}$ a possibly empty set of indices. The nonlinearity of the above system is expressed by the boolean functions $b_{i l}: \mathbb{R}_{+}^{n} \rightarrow\{0,1\}$ defined in terms of sums and multiplications of step functions, $s^{+}$ and $s^{-}$given by

$$
s^{+}\left(x_{j}, \theta_{j}\right)=\frac{1+\operatorname{sgn}\left(x_{j}-\theta_{j}\right)}{2} ; \quad s^{-}\left(x_{j}, \theta_{j}\right)=1-s^{+}\left(x_{j}, \theta_{j}\right),
$$

for some threshold $\theta_{j}>0$. They specify the conditions under which the gene $i$ is expressed at a rate $k_{i l}$. System (48) has been widely studied in the literature, mainly in the framework of genetic networks (see [32] and references therein). In vector notations, (48) rewrites as

$$
\dot{x}=-\gamma x+\sum_{l \in L} k_{l} \prod_{j=1, n}\left(1+c_{j}^{l} \operatorname{sgn}\left(x_{j}-\theta_{j}^{l}\right)\right),
$$

where $\gamma=\operatorname{diag}\left(\gamma_{1}, \ldots, \gamma_{n}\right), c_{j}^{l} \in\{-1,0,1\}, L$ counts all products of step functions in the definition of $k_{i l}, l \in L_{i}, i=1, \ldots, n$ and for each $l \in L, k_{l} \in \mathbb{R}^{n}$. The use of step functions allows one for a compact description of the dynamics of genetic regulatory networks, based on differential inclusions. In this case Filippov framework is employed ([33], [40], [43]).

An alternative method in the study of (48) is based on replacing step functions by special smooth functions, namely sigmoids (also called "logoids") and investigating the system thus obtained. The main technical tool consists in analyzing the limit when all sigmoids approach step functions. In [64], it is shown that in this case the solutions for sigmoids approach the limit solution uniformly in a finite time. A complete mathematical comparison between these two approaches has been featured recently [56].

\section{Complementarity systems}

In this section we treat the case when $K \subseteq \mathbb{R}^{l}$ in (13) is a nonempty closed convex cone. Proposition 1.1.3 in [38] establishes an equivalence between the variational problem given by the inequality (13) and the conewise complementarity problem, denoted $C C P(K)$, to follow: for each $t \geq 0$, find $\lambda(t) \in \mathbb{R}^{l}$ such that

$$
K \ni \lambda(t) \perp y(t) \in K^{*} .
$$


Hereafter we will consider linear (or affine) conewise complementarity systems (CCS) of the form:

$$
\left\{\begin{array}{l}
\dot{x}(t)=A x(t)+B \lambda(t)+a \\
K \ni \lambda(t) \perp y(t)=C x(t)+c+D \lambda(t) \in K^{*} \\
x(0)=x_{0} \in \mathbb{R}^{n}
\end{array}\right.
$$

Let us present some examples which illustrate the relevance of the study of CCS.

Example 8.1. (mass and spring/dashpot, relative degree 1). Let us come back to Example 3.2. Let us introduce $\xi$ as the spring/dashpot coordinate (its deformation), and define $x^{T}=(q, \dot{q}, \xi)$. We write the dynamics as [12, Example 3]:

$$
\left\{\begin{array}{l}
\dot{x}(t)=A x(t)+B \lambda(t) \\
0 \leq y(t)=C x(t) \perp \lambda(t) \geq 0
\end{array}\right.
$$

with $A=\left(\begin{array}{ccc}0 & 1 & 0 \\ 0 & 0 & 0 \\ 0 & 0 & -\frac{k}{c}\end{array}\right), B^{T}=\left(\begin{array}{lll}0 & \frac{1}{m}-\frac{1}{c}\end{array}\right), C=\left(\begin{array}{lll}1 & 0 & -1\end{array}\right) ; \lambda(t)$ has the physical interpretation of a contact force. There are two modes: if $y(t)>0$ then $\lambda(t)=0$ and $\ddot{q}(t)=0$. If $y(t)=0$ on some time interval $\left[t_{0}, t_{1}\right], t_{0}<t_{1}$, then the complementarity conditions are rewritten in "velocity" [42] [2, Propositions C.8, C.9]: $0 \leq \dot{y}(t)=$ $C A x(t)+C B \lambda(t) \perp \lambda(t) \geq 0$, and since $C B=\frac{1}{c}>0$ this LCP always has a unique solution. This is found by inspection to be $\lambda(t)=0$ if $\dot{q}(t)+\frac{k}{c} q(t)>0$, and $\lambda(t)=-c \dot{q}(t)-k q(t)$ if $\dot{q}(t)+\frac{k}{c} q(t) \leq 0$.

The switching conditions are rather different from those in Example 3.2. Such a system has a relative degree one between $\lambda$ (the "input") and $y$ (the "output"). This model guarantees that the contact force keeps the right sign for all times. The kinematic restitution coefficients that result from (3) and from (52) are quite different one from each other [5]. Obviously we can rewrite (52) as the differential inclusion

$$
-\dot{x}(t)+A x(t) \in B N_{K}(C x(t))
$$

with $K=\mathbb{R}^{+}$. The same comments as for Example 6.4 apply: this is not a switching system as in (2). When $c=0$ however both systems are the same because the contact force is equal to $-k q$ whose sign is the same as the signed distance between the obstacle and the mass.

Example 8.2. (RLC circuit with ideal diode, relative degree 0) Let us consider the circuit in Figure $5(a)$, where the diode is an ideal diode [3, 4]. Its dynamics is given by:

$$
\left\{\begin{array}{l}
\dot{x}_{1}(t)=x_{2}(t)-\frac{1}{R C} x_{1}(t)-\frac{\lambda(t)}{R} \\
\dot{x}_{2}(t)=-\frac{1}{L C} x_{1}(t)-\frac{\lambda(t)}{L} \\
0 \leq \lambda(t) \perp y(t)=\frac{\lambda(t)}{R}+\frac{1}{R C} x_{1}(t)-x_{2}(t) \geq 0
\end{array}\right.
$$

where $x_{1}$ is the charge of the capacitor and $x_{2}$ is the current through the inductor. The signal $y$ depends directly on $\lambda$, so the relative degree between them two is 0 . This systems belongs to the class (2) and the switching surface is easily identified as $\frac{1}{R C} x_{1}-x_{2}=0$. The vector field is continuous, which means that the criteria of Section 4 are satisfied. 


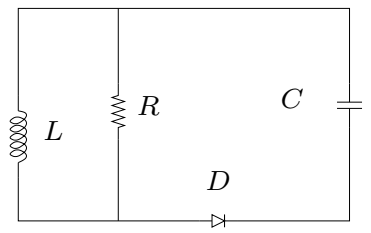

(a)

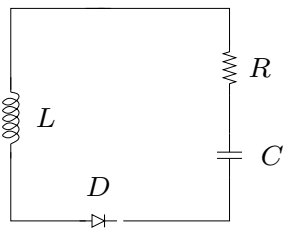

(b)

Figure 5: Electrical circuits with capacitors, resistors and ideal diodes.

Example 8.3. (RLC circuit with ideal diode, relative degree 1). Let us consider the circuit in Figure $5(b)$, where the diode is an ideal diode. Its dynamics is given by:

$$
\left\{\begin{array}{l}
\dot{x}_{1}(t)=x_{2}(t) \\
\dot{x}_{2}(t)=-\frac{R}{L} x_{2}(t)-\frac{1}{L C} x_{1}(t)-\frac{\lambda(t)}{L} \\
0 \leq \lambda(t) \perp y(t)=-x_{2}(t) \geq 0
\end{array} .\right.
$$

The signal y does not depend directly on $\lambda$, however its time derivative along the system's trajectories does. The relative degree is equal to 1 .

When $K=\mathbb{R}_{+}^{l}$, the linear CCS (51) results in what is simply called a Linear Complementarity System (LCS):

$$
\left\{\begin{array}{l}
\dot{x}(t)=A x(t)+B \lambda(t) \\
0 \leq \lambda(t) \perp y(t)=C x(t)+c+D \lambda(t) \geq 0 \\
x(0)=x_{0} \in \mathbb{R}^{n} .
\end{array}\right.
$$

If $D$ is a $P$-matrix, a well-known result from complementarity theory states that $\lambda(t)$ is a piecewise-linear function of $C x(t)+c$ [27]. Thus, (56) is an ordinary differential equation with Lipschitz continuous right-hand side and $\mathcal{C}^{1}$ solutions. A general rewriting of the LCS in (56) with $c=0$ and $D$ a $P$-matrix, as a switching system (2) is given in [22, Equation (2.17)]. The switching surfaces however appear only in an implicit way, because for a generic $P$-matrix it becomes rapidly impossible to get an explicit description of the different modes of the LCP. In the next example the cells of a planar complementarity systems are explicitly described, for a non trivial $D$ matrix.

Example 8.4. Consider (in the plane) the complementarity condition in (56) with $C=$ $I_{2}, c=0$ and $D=\left(\begin{array}{ll}2 & 1 \\ 1 & 1\end{array}\right)$ (so D is a P-matrix). The following cases are in range.

i) $\lambda_{1}, \lambda_{2}>0$ and $x_{1}+2 \lambda_{1}+\lambda_{2}=0, x_{2}+\lambda_{1}+\lambda_{2}=0$. Then, the complementarity problem has the unique solution $\lambda=\left(x_{2}-x_{1}, x_{1}-2 x_{2}\right)^{T}$ if $x \in R_{1}$ where $R_{1}=\left\{x \in \mathbb{R}^{2}: x_{1}<0, x_{2} \in\left(x_{1}, \frac{x_{1}}{2}\right)\right\}$.

ii) $\lambda_{1}>0, \lambda_{2}=0$ and $x_{1}+2 \lambda_{1}+\lambda_{2}=0, x_{2}+\lambda_{1}+\lambda_{2} \geq 0$. The complementarity problem has the unique solution $\lambda=\left(-\frac{x_{1}}{2}, 0\right)^{T}$ if $x \in R_{2}$ where $R_{2}=\left\{x \in \mathbb{R}^{2}\right.$ : $\left.x_{1}<0, x_{2} \geq \frac{x_{1}}{2}\right\}$.

iii) $\lambda_{1}=0, \lambda_{2}>0$ and $x_{1}+2 \lambda_{1}+\lambda_{2} \geq 0, x_{2}+\lambda_{1}+\lambda_{2}=0$. The complementarity problem has the unique solution $\lambda=\left(0,-x_{2}\right)^{T}$ if $x \in R_{3}$ where $R_{3}=\left\{x \in \mathbb{R}^{2}\right.$ : $\left.x_{2}<0, x_{1} \geq x_{2}\right\}$. 
iv) $\lambda_{1}=\lambda_{2}=0$ and $x_{1}+2 \lambda_{1}+\lambda_{2} \geq 0, x_{2}+\lambda_{1}+\lambda_{2} \geq 0$. The complementarity problem has the unique solution $\lambda=(0,0)^{T}$ if $x \in R_{4}$ where $R_{4}=\left\{x \in \mathbb{R}^{2}\right.$ : $\left.x_{1}, x_{2} \geq 0\right\}$.

It is easy to see that $x \mapsto \lambda(x)$ is piecewise linear (thus, continuous) and there are four regions that can be described like in Section 3.1, where the system is completely defined (see Figure 6). So, for any $A, B \in \mathcal{M}_{2,2}(\mathbb{R})$, system (56) admits a unique classical solution and thus, the continuity obtained in the right-hand side of system (56) allows for weak instead of strict inequalities in the final description of the regions.

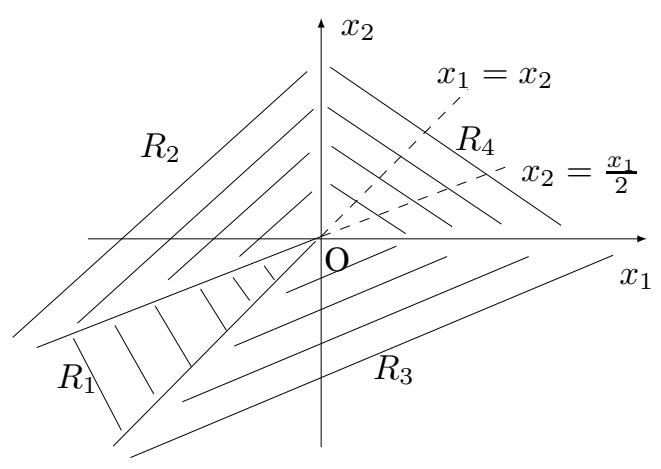

Figure 6: The four regions in Example 8.4.

Let us denote by $\operatorname{Sol}(C x, c, D)$ the solution set of the complementarity problem given by the complementarity condition in (56). It follows from [21, Proposition 2.1] that the LCS has a unique $\mathcal{C}^{1}$ solution if and only if $\operatorname{Sol}(C x, c, D)$ is a singleton for all $x \in \mathbb{R}^{n}$. More generally, in [38] it is shown that in the case when $K$ is a polyhedral cone, the set of all solutions of the complementarity problem:

$$
K \ni \lambda(t) \perp y(t)=C x(t)+c+D \lambda(t) \in K^{*}
$$

is a singleton if the following conditions are satisfied:

i) $D$ is positive semidefinite (possibly non symmetric),

ii) $C \mathbb{R}^{n} \subseteq-D K+K^{*}$,

iii) $(K-K) \cap K \operatorname{Ker}\left(D+D^{T}\right) \subset \operatorname{Ker}(B)$.

From (i) it follows that the LCS (56) has a piecewise linear right-hand side, and this corresponds to a uniform relative degree 0 between $\lambda$ and $y$ (if in addition $D=D^{T}$ then one may apply (23) with $\left.K=\mathbb{R}_{+}^{l}\right)$. From (57) one has that $\lambda(t) \in(D$. $\left.+N_{K}\right)^{-1}(-C x(t)-c)$ so that i), ii) and iii) guarantee that this operator is singlevalued. In a more general setting, Proposition 5.1 and Theorem 5.1 in [61] provide quite general conditions such that the variational inequality possesses a unique solution (Lipschitz continuous) so that the dynamical system (a differential variational inequality in [61], an AVS in this paper) has a unique $\mathcal{C}^{1}$ solution. These results in fact provide conditions under which the operator $B\left(D \cdot+N_{K}\right)^{-1}(-C x-c)$ is single-valued, so that (29) is an ordinary differential equation.

In the case when $D=0$ but $C B$ has full-rank, the relative degree is equal to 1 . The complementarity systems in Examples 8.1 and 8.3 have a relative degree 1. As we have seen in Example 6.4 such an LCS cannot be recast into (2). We therefore do 
not insist on this case, but it is worth noting that the relative degree one is a common case for physical systems like mechanical or electrical systems, showing the limitations of the models as discontinuous systems as in (2). Let us mention anyway that using the results in [17] or [16] one may prove the existence and uniqueness of absolutely continuous, or Lipschitz continuous, solutions in the relative degree one case. This suggests that the regularity (or the smoothness) of the solutions is intimately linked to the relative degree between the multiplier $\lambda$ (playing the role of an input signal) and the complementarity variable $y$ (playing the role of an output signal). This is indeed the case, see [6] for more details.

Finally, we notice that relay systems may also be recast into complementarity systems, since the sign multifunction in Figure 2 (a) lends itself to a description via complementarity, see $e . g$. [2, Chapter 1]. This is in fact a particular case of representing a piecewise linear multifunction (vertical segments are admitted) into a complementarity framework [53]. See Section 12.

\section{Time-varying switching systems}

The frameworks of relay and of complementarity systems allow one to consider in a rather natural way the case of nonlinear and/or time-varying vector fields $f_{i}$ in (2) and of time-varying cells $\chi_{i}(t)$. For instance [19] considers linear complementarity systems of the form:

$$
\left\{\begin{array}{l}
\dot{x}(t)=A x(t)+B \lambda(t)+g(t) \\
0 \leq y(t)=C x(t)+D \lambda(t)+h(t) \perp \lambda(t) \geq 0
\end{array}\right.
$$

where $(A, B, C, D)$ is supposed to be a dissipative (or positive real) quadruplet ([15]). When $D=0$ this implies in particular that an input/output constraint $P B=C^{T}$ as in Theorem 7.11 is satisfied. Otherwise the dissipative linear matrix inequality implies $D+D^{T} \geq 0$. Notice that both the smooth dynamics in Examples 8.2 and 8.3 are dissipative. Theorem 7.5 in [19] states conditions under which (58) has a unique global solution $(x, \lambda, y)$ where $g$ and $h$ are so-called piecewise Bohl functions. The solutions are the sum of functions (regular terms) in the extended $L_{l o c}^{2}$ space, and Dirac measures (impulsive terms). It is noted in [19] that the jumps in the state $x$ show up only at the times where $h$ is discontinuous (such a fact is explained also in [16] in the context of measure differential inclusions, where a rigorous meaning of the complementarity conditions at the times of state jumps is provided).

Let $D$ be a $P$-matrix, it is clear that (58) can be interpreted as a switching system (2) with time-varying vector fields and cells.

Example 9.1. Let us consider the scalar system with $d>0$ :

$$
\left\{\begin{array}{l}
\dot{x}(t)=a x(t)+b \lambda(t)+g(t) \\
y(t)=C x(t)+c+d \lambda(t)+h(t) . \\
0 \leq \lambda(t) \perp y(t) \geq 0
\end{array} .\right.
$$

The multiplier $\lambda(t)$ is the solution of the LCP: $0 \leq C x(t)+c+d \lambda(t)+h(t) \perp \lambda(t) \geq 0$, that is given by: $\lambda(t)=0$ if $C x(t)+c+h(t)>0$ and $\lambda(t)=-C x(t)-c-h(t)$ if $C x(t)+c+h(t) \leq 0$. The system in (59) is therefore equivalent to the switching system:

$$
\dot{x}(t)= \begin{cases}a x(t)+g(t) & \text { if } C x(t)+c+h(t)>0 \\ (a-b C) x(t)+f(t) & \text { if } C x(t)+c+h(t)<0\end{cases}
$$

RR $n^{\circ} 7760$ 
where $f(t)=-b c-b h(t)+g(t)$, that is a switching system with time-varying switching surface $\Sigma_{t}=\{z \in \mathbb{R}: C z+c+h(t)=0\}$.

It is easy to add some current or voltage sources in the circuits in Figure 5 so that (54) and (55) become time-varying. The relay systems as in (34) also form a class of non-autonomous switching systems, whose cells do not vary but whose vector fields $f_{i}$ do. The time-varying linear complementarity systems (58) have also been studied in [16]. The overall framework in [16] is that of the perturbed Moreau's sweeping process, that is a specific differential inclusion into normal cones to time-varying convex sets $K(t)$, roughly: $-\dot{x}(t)+g(x(t), t) \in N_{K(t)}(x(t))$. The main assumption is that there exists $P=P^{T}>0$ such that $P B=C^{T}$. Then a state space transformation as in Theorem 7.11 is done to recast (58) into a differential inclusion of the form (when $D=0)$ :

$$
-\dot{z}(t)+R A R^{-1} z(t)+R g(t) \in N_{S(t)}(z(t)) .
$$

The time-variation of the convex polyhedral set $S(t)$ is due solely to the term $h(t)$ in (59). The solutions are absolutely continuous when $S(t)$ is (as a set), and of local bounded variation when $S(t)$ is (as a set). In the latter case solutions may jump and the differential inclusion (61) has to be rewritten as a measure differential inclusion. The case $D \geq 0$ is alluded to in [16]. This case is however more deeply treated in [17], but with $h(t)=0$ (hence one is no longer in the framework of Moreau's sweeping process because the underlying convex set within which the system's state evolves, becomes constant). The solutions are then continuous (see Section 10).

\section{Multivalued Lur'e dynamical systems}

It happens that all the foregoing systems (relay, complementarity) possess a very strong underlying structure of a continuous, single-valued system with a feedback interconnection that consists of a multivalued, static (i.e. independent of the state), possibly time-varying, nonlinearity. Such a point of view is obvious from (12)-(13). This makes such systems much more structured than the general switching systems (2). The point of view of Lur'e dynamical nonsmooth, multivalued systems is taken in [13, 17]. Therein one starts from a formalism that is close to the one in (12)-(13) and reads as follows. Let $A: \mathbb{R}^{n} \rightarrow \mathbb{R}^{n}$ be a (possibly) nonlinear operator, $B \in \mathcal{M}_{n, p}(\mathbb{R})$, $C \in \mathcal{M}_{p, n}(\mathbb{R})$ and $D \in \mathcal{M}_{p, p}(\mathbb{R})$ given matrices, $f: \mathbb{R}_{+} \rightarrow \mathbb{R}$ continuous such that $\dot{f} \in L_{\text {loc }}^{1}\left(\mathbb{R}_{+} ; \mathbb{R}^{n}\right)$ and $\Phi: \mathbb{R}^{p} \rightarrow \mathbb{R} \cup\{+\infty\}$ a given proper convex and lower semicontinuous function (see [66]). Let $x_{0} \in \mathbb{R}^{n}$ be some initial condition, we consider the problem: Find $x: \mathbb{R}_{+} \rightarrow \mathbb{R}^{n}$ continuous such that $\dot{x} \in L_{\text {loc }}^{\infty}\left(\mathbb{R}_{+} ; \mathbb{R}^{n}\right)$ and $x$ right-differentiable on $\mathbb{R}_{+}, \lambda: \mathbb{R}_{+} \rightarrow \mathbb{R}^{p}$ continuous and $y: \mathbb{R}_{+} \rightarrow \mathbb{R}^{p}$ continuous satisfying the nonsmooth Lur'e system $N S L S\left(A, B, C, D, f, \Phi, x_{0}\right)$ :

$$
\left\{\begin{array}{l}
\dot{x}(t)=A(x(t))+B \lambda(t)+f(t), \text { a.e. } t \geq 0 \\
y(t)=C x(t)+D \lambda(t), t \geq 0 \\
\lambda(t) \in-\partial \Phi(y(t)), t \geq 0 \\
x(0)=x_{0}
\end{array} .\right.
$$

Two paths are followed in [17]. The first one consists of considering that $D \geq 0$ has the structure $\operatorname{diag}\left(D_{I}, 0\right)$ with $D_{I} \geq 0$, and to transform the system (62) into a suitable variational inequality so that Kato's theorem (or one of its variant, see [52]) applies directly. Roughly, this uses the fact that the part of $y$ that does not depend on $\lambda$ 
corresponds to a multivalued nonlinearity, whereas the part that depends on $\lambda$ via $D_{I}$ defines a single valued operator (in a way quite similar to what happens in Example 6.3). The single-valued part of the feedback interconnection is therefore incorporated into the single-valued part of the system, i.e. it is added to $A(x)$ ), while the feedback interconnection is left with only the multivalued part.

The second path uses in fact the rewriting of the system using an operator as the one in (28). Using convex analysis tools one may invert the inclusion in (62) so that it rewrites:

$$
\left\{\begin{array}{l}
\dot{x}(t)=A(x(t))+B \lambda(t)+f(t), \text { a.e. } t \geq 0 \\
y(t)=C x(t)+D \lambda(t), t \geq 0 \\
y(t) \in-\partial \Xi(\lambda(t)), t \geq 0 \\
x(0)=x_{0}
\end{array}\right.
$$

for some convex, proper, lower semicontinuous function $\Xi$ that is obtained from $\Phi$ by an inversion process similar to the one used in Example 6.2, i.e. $\Xi(z)=\Phi^{*}(-z)$ (the minus sign is here to preserve the minus sign in the feedback interconnection in (63)). The Lur'e system structure clearly appears in (63). By properly choosing $\Phi$ (hence $\Xi$ ) one may recover some classes of nonlinear conewise complementarity system $\left(\Phi=\Psi_{K}\right.$ for some closed, non empty convex cone $\left.K\right)$ and of nonlinear relay systems $\left(\Phi(y)=\left|y_{1}\right|+\ldots+\left|y_{l}\right|\right)$. It is clear from (63) that the crucial operator for this differential inclusion is $x \mapsto B(D \cdot+\partial \Xi)^{-1}(-C x) \ni \lambda$. The works in [17] aim at characterizing it accurately depending on $D$ and $\Xi$. For instance, it follows from Corollary 1 in [17] that this operator is single-valued and Lipschitz continuous when $\Phi=\Psi_{K}$ with $K$ a closed convex cone if (compare with the conditions below (57)):

(i) $D$ is positive semidefinite (possibly non symmetric),

(ii) $K^{o} \cap \operatorname{Ker}\left(D+D^{T}\right)=\{0\}$,

(iii) $\operatorname{Im}(C) \subset \operatorname{Im}\left(D+D^{T}\right) \subset \operatorname{Ker}(B)$,

where $K^{o}$ is the polar cone of $K$. Other criteria that guarantee that the fundamental operator is single-valued are given in Section 3 of [17].

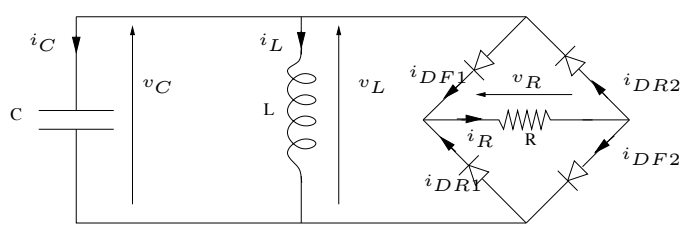

Figure 7: A 4-diode bridge wave rectifier.

Example 10.1. Let us consider the four-diode bridge wave rectifier in figure 7, with a capacitor $\mathbf{C}>0$, an inductance $\mathbf{L}>0$, a resistor $\mathbf{R}>0$. Its dynamics is given by:

$$
\begin{gathered}
{\left[\begin{array}{c}
\dot{x}_{1}(t) \\
\dot{x}_{2}(t)
\end{array}\right]=\left[\begin{array}{cc}
0 & -\frac{1}{\mathrm{C}} \\
\frac{1}{\mathrm{~L}} & 0
\end{array}\right]\left[\begin{array}{l}
x_{1}(t) \\
x_{2}(t)
\end{array}\right]+\left[\begin{array}{cccc}
0 & 0 & -\frac{1}{\mathrm{C}} & \frac{1}{\mathrm{C}} \\
0 & 0 & 0 & 0
\end{array}\right] \lambda(t)} \\
0 \leq y(t) \perp \lambda(t) \geq 0
\end{gathered}
$$


where $x_{1}=v_{L}, x_{2}=i_{L}, \lambda=\left(-v_{D R 1}-v_{D F 2} i_{D F 1} i_{D R 2}\right)^{T}, y=\left(i_{D R 1} i_{D F 2}-\right.$ $\left.v_{D F 1}-v_{D R 2}\right)^{T}$ and

$$
y=\left[\begin{array}{cc}
0 & 0 \\
0 & 0 \\
-1 & 0 \\
1 & 0
\end{array}\right]\left[\begin{array}{l}
x_{1} \\
x_{2}
\end{array}\right]+\left[\begin{array}{cccc}
\frac{1}{\mathrm{R}} & \frac{1}{\mathrm{R}} & -1 & 0 \\
\frac{1}{\mathrm{R}} & \frac{1}{\mathrm{R}} & 0 & -1 \\
1 & 0 & 0 & 0 \\
0 & 1 & 0 & 0
\end{array}\right] \lambda
$$

Notice that in this example one has $n=2$ and $l=4$. The matrix $D$ is full rank, semidefinite positive. The relation $P B=C^{T}$ holds with $P=\left(\begin{array}{cc}\mathbf{C} & 0 \\ 0 & p_{22}\end{array}\right), p_{22}>0$, where $\mathbf{C}>0$ is the capacitor parameter. This example shows that considering $D \geq 0$ (and not $D>0$ nor $D=0$ ) is important for applications.

The relative degree one systems in Examples 8.1 and 8.3 can also be analyzed with the tools developed in [17]. The approach in [17] strongly relies on a "dissipative input/output" constraint similar to the one of Theorem 7.11, i.e. there exists $P=P^{T}>$ 0 such that $P B=C^{T}$. The matrix $D$ is supposed to be positive semi-definite. The underlying property is the maximal monotonicity of the multivalued part of the system.

Remark 10.2. Electrical circuits as in example 10.1 show that non symmetric matrices are common in complementarity systems. It is noteworthy that the system in (64) has a full rank $D$ that is only semi positive definite, because of its non zero skew-symmetric part. This shows that the relative degree $r=0$ is not sufficient by itself to guarantee that the LCP is well-posed. In the multivariable case we have that $D>0 \Rightarrow r=0$, but $r=0$ does not imply $D>0$ (it does if $D=D^{T}$ ).

Example 10.3. Let us consider two masses moving on a line, linked by a constant spring with stiffness $k$ (possibly nonlinear), subject to Coulomb friction with friction coefficients $\mu_{1}>$ and $\mu_{2}>0$, and acted upon by two external forces $F_{1}$ and $F_{2}$. The dynamics is given by:

$$
\left\{\begin{array}{l}
m_{1} \ddot{q}_{1}(t) \in-m_{1} \mu_{1} g \operatorname{Sgn}\left(\dot{q}_{1}(t)\right)+k\left(q_{2}(t)-q_{1}(t)\right)+F_{1}(t) \\
m_{2} \ddot{q}_{2}(t) \in-m_{2} \mu_{2} g \operatorname{Sgn}\left(\dot{q}_{2}(t)\right)+k\left(q_{1}(t)-q_{2}(t)\right)+F_{2}(t)
\end{array}\right.
$$

The subspace $\left\{x \in \mathbb{R}^{4}: \dot{q}_{1}=\dot{q}_{2}=0\right\}$ where $x=\left(\begin{array}{lll}q_{1} & \dot{q}_{1} & q_{2} \\ \dot{q}_{2}\end{array}\right)^{T}$ represents a codimension 2 attractive surface. The well-posedness of this relay system may be stated using Theorem 7.11 or the results in [17]. There exists $P=P^{T}>0$ such that $P B=C^{T}$, where $B$ and $C$ are easily identified from (66): $P=\operatorname{diag}\left(p_{i i}\right)$ with $p_{11}>0, p_{33}>, p_{22}=\frac{1}{g \mu_{1}}, p_{44}=\frac{1}{g \mu_{2}}$. More examples such mechanical systems with one-dimensional Coulomb friction may be found in [8], where they are analyzed via maximal monotone differential inclusions.

Example 10.4. Consider now the system made of two masses $m_{1}$ and $m_{2}$, with $m_{1}$ sliding on the top of $m_{2}$ while $m_{2}$ is in contact with the ground. The coefficients of friction are $\mu_{1}>0$ between the two masses, and $\mu_{2}>0$ between $m_{2}$ and the ground. The dynamics is given by:

$$
\left\{\begin{aligned}
m_{1} \ddot{q}_{1}(t) & \in-m_{1} \mu_{1} g \operatorname{Sgn}\left(\dot{q}_{1}(t)-\dot{q}_{2}(t)\right) \\
m_{2} \ddot{q}_{2}(t) & \in m_{1} \mu_{1} g \operatorname{Sgn}\left(\dot{q}_{1}(t)-\right. \\
& \left.-\dot{q}_{2}(t)\right)-\left(m_{1}+m_{2}\right) g \mu_{2} \operatorname{Sgn}\left(\dot{q}_{2}(t)\right)
\end{aligned}\right.
$$


This system is compactly rewritten as $\dot{x}(t) \in-B \operatorname{Sgn}(C x(t))$, with $C=\left(\begin{array}{cc}1 & -1 \\ 0 & 1\end{array}\right)$, $B=\left(\begin{array}{cc}\mu_{1} g & 0 \\ -\frac{m_{1}}{m_{2}} \mu_{1} g & \frac{m_{1}+m_{2}}{m_{2}} \mu_{2} g\end{array}\right)$. There does not exist $P=P^{T}>0$ such that $P B=$ $C^{T}$, except if $\frac{m_{1}+m_{2}}{m_{1}}=\frac{\mu_{1}}{\mu_{2}}$. However the transfer matrix

$$
G(s)=\frac{1}{s}\left(\begin{array}{cc}
\mu_{1} g\left(1+\frac{m_{1}}{m_{2}}\right) & -\frac{m_{1}+m_{2}}{m_{2}} \mu_{2} g \\
-\frac{m_{1}}{m_{2}} \mu_{1} g & \frac{m_{1}+m_{2}}{m_{2}} \mu_{2} g
\end{array}\right)
$$

is a $P$-matrix since all its principal minors (there are three) are positive. Therefore Theorem 7.11 does not apply, neither the results in [17], but Theorem 7.5 applies.

\section{Summary and comments}

From the above results summarized in Sections 7 to 10, it follows that the main tools and assumptions that have been employed to study the AVS (12)-(13) are:

- Complementarity theory and the $P$ property of matrices or functions,

- Maximal monotonicity of multivalued operators,

- Dissipativity of dynamical systems.

The fundamental operator for the analysis of the AVS in (12)-(13) is

$$
\Phi: x \mapsto B\left(D \cdot+N_{K}\right)^{-1}(-C x-c)
$$

whose properties depend mainly on $D$ and $K$. It may be single-valued (e.g. $D=D^{T}>$ 0 and $K$ a convex set) or multivalued (e.g. $D$ a $P_{0}$-matrix and $K$ a closed rectangle). The functional spaces for the solutions vary from one result to the other: $\mathcal{C}^{1}$, absolutely continuous, Lipschitz continuous, $L_{2}$, of local bounded variations, locally or piecewise analytic. The advantage of the AVS (12)-(13) over (2) is that it provides compact formalisms with a strong structure that are very suitable for mathematical analysis, time-discretization, and stability analysis. For instance, they allow the introduction of time-varying cells, of nonlinearities, and they are more tractable for proving uniqueness using powerful tools of convex, complementarity, or nonsmooth analysis. Determining the continuity of the underlying vector field $f$ may be done via high-level tools like (23) that dispenses one with examining each vector field $f_{i}$ at each cell boundary as it is done in Section 4. On the contrary, the structure of a general switching system as (2) is quite loose. It is worth noticing that the monotonicity (and its extensions like onesided Lipschitz continuity) is a very important property for proving the uniqueness of solutions. However, in [26], there are identified other sufficient conditions specifically tailored for piecewise continuous systems. The great advantage of all the techniques based on maximal monotonicity is that they allow one to consider non-linearities.

Another fundamental parameter in AVS is the relative degree between $\lambda$ and $y$. The relative degree influences the uniqueness of solutions in relay systems (see Section 7.3) and the smoothness of the solutions in linear complementarity systems (see [6]). In this setting the dissipativity property is quite useful since a positive real quadruplet $(A, B, C, D)$ has $D+D^{T} \geq 0$ and, if $D=0$, satisfies a constraint of the form $P B=C^{T}$ with $P=P^{T}>0$. 
- The various sets which play a role in the above developments (the cells $\chi_{i}$, the sets $K$ for the AVS) are all convex. Convexity therefore appears to be a central property (the positive definiteness of the matrix $D$ may also be interpreted as a convexity property). Convexity is in fact closely related to the maximal monotonicity and to the dissipativity. Indeed when $K$ is a closed convex non empty set, then the mapping $\lambda \mapsto N_{K}(\lambda)$ is maximal monotone. The generalized equation in (33) has a unique solution when $D$ is positive definite and $K$ is closed convex non empty. More generally the well-posedness of the differential inclusion $\dot{x}(t) \in \Phi(x(t))$ relies heavily on convexity properties. The role of dissipativity-like properties and their link to convexity is highlighted in Theorem 7.11 , where the chain rule for convex functions plays a central role. Whether or not all the material that is presented in this paper extends to non convex sets, is an interesting question. Starting from the point of view of inclusions into normal cones as in (29) (or of AVS in (12) and (13)), a natural extension is that of prox-regular sets $[16,25]$. Another closely related important point is that the argument of the sign multifunction for relay systems, and the variable $y(t)$ for complementarity systems, have been considered as linear (or affine) functions of $x$ and $\lambda$. This means that the associated sets $\chi_{i}$ in the switching system formalism, are convex. Thus starting from prox-regular AVS might help in defining well-posed switching systems with non convex cells $\chi_{i}$.

- Another interesting point is to investigate how the dissipativity and monotonicity properties used in the framework of AVS (relay, complementarity systems) relate to dissipativity of switching systems as in [76]. The AVS framework allows one to state the dissipativity with a unique supply rate and a unique storage function (more precisely, a unique passivity linear matrix inequality [15, Chapter 3]), whereas the criterion in [76] uses several supply rates and storage functions. This may constitute a strong advantage of working within the AVS framework, when this is possible, and paves the way towards extensions of feedback controllers synthesis as in [35]. Observer design for classes of set-valued systems using dissipativity has been proposed in [18] and [47]. The applications in the stability and the feedback control of nonsmooth electro-mechanical systems and circuits seems to be a promising field of research.

- Switching feedback controllers formulated through complementarity conditions have not yet received much attention, except in [51]. The parameter identification of nonsmooth systems using multiple relay functions is also a topic that deserves attention [24]. The results on relay and complementarity systems may be used as a theoretical foundation for the design of such inputs and identfication techniques. The relay system used in [24, Equ.(7)-(10)] fits with (30), however Theorems 7.5 and 7.11 do not apply. This system is similar to the so-called twisted controller of sliding-mode control, for which specific stability results have been developed that relax the uniqueness of solutions [60, Chapter 3].

- It is known in circuit theory that feedback controllers implemented through current or voltage sources may increase the relative degree (the index when one remains within the DAE framework). The relative degree influences the uniqueness of solutions in relay systems (see Section 7.3) and the smoothness of the solutions in linear complementarity systems (see [6]). Stability, control and simulation of nonsmooth circuits with higher relative degree is still a largely open field. 
- The properties of finite-time convergence, which are a particular feature of nonsmooth systems $[59,60]$, can certainly be used in a more systematic way to refine the well-posedness results. Indeed in many instances the solutions are "more than absolutely continuous", as stated by general results on differential inclusions. This is especially true for set-valued Lur'e dynamical systems as in Section 10 .

- The control of relay systems in biology and gene regulatory networks (see Section 7.5) is a topic with promising applications, see [39].

As announced above in this survey only systems with continuous solutions are dealt with. State jumps may occur in AVS when some unilateral effects are present (state inequality constraints), like in complementarity systems with $D=0$. Roughly speaking state jumps may occur each time the domain of the operator in (68) is not the whole of $\mathbb{R}^{n}$, and $x\left(t^{-}\right)$does not belong to this domain at some $t$. Then the state has to jump to some admissible value $x\left(t^{+}\right)$. There are two issues with state jumps: (i) formulate a coherent state jump law (in Contact Mechanics this belongs to the realm of impact modeling, for electrical circuits see [41])), (ii) correctly rewrite the dynamics, since the solutions usually no longer are functions but distributions (see [6] for a complete study of a class of distribution differential inclusions that extend Moreau's measure differential inclusions $[57,58])$. Notice that the fundamental operator may be multivalued but with no unilateral effects, as in relay systems. On the contrary unilaterality implies some kind of multivaluedness.

Finally let us point out that nonsmooth systems like AVS may be recast in the class of so-called "hybrid dynamical systems", see e.g. [12,22]. This approach is used in [22] to determine when a conewise switching system (the cells $\chi_{i}$ are cones, i.e. $h_{j}=0$ in the definition of $d_{j}$ ) undergoes at most a finite number of switches in finite time (non Zeno behaviour).

\section{From switching systems to AVS}

In the previous sections we have analyzed several classes of nonsmooth systems (relay and complementarity systems) which are, under certain conditions, switching systems as in (2). Let us now make the inverse process: is it possible to construct an AVS from (2)? The answer is yes in some particular cases. Such an issue is closely related to finding the representation as a complementarity problem, of a piecewiselinear function. Let us study the simplest case of a switching system with switching surface $\Sigma=\left\{x \in \mathbb{R}^{n}: H x+h=0\right\}$ that separates the state space in two cells $\chi_{1}=\left\{x \in \mathbb{R}^{n}: H x+h>0\right\}$ and $\chi_{2}=\left\{x \in \mathbb{R}^{n}: H x+h<0\right\}$. The two vector fields are $A_{1} x+a_{1}$ and $A_{2} x+a_{2}$, and we suppose that the continuity holds on $\Sigma$, so that the conditions of Theorem 4.4 hold true. It is then not difficult to see that for $B$ and $D$ such that $v_{1,2}=\frac{B}{D}$ with $D>0$ (for instance, $D=|h|$ or even more $D=1$ and $B=v_{1,2}$ ), the LCS:

$$
\left\{\begin{array}{l}
\dot{x}(t)=A_{1} x(t)+a_{1}+B \lambda(t) \\
0 \leq \lambda(t) \perp H x+h+D \lambda(t) \geq 0
\end{array}\right.
$$

is the complementarity representation of the switching system. Indeed when $H x+h>$ 0 then $\lambda=0$, when $H x+h<0$ then $\lambda=-\frac{1}{D}(H x+h)$ and the vector field is $\left(A_{1}-\frac{1}{D} B H\right) x+a_{1}-\frac{1}{D} B h$ that is equal to $A_{2} x+a_{2}$, by Theorem 4.4. It is 
possible to extend this to more complex switching systems. This has been tackled in $[53,72]$. For instance, all continuous piecewise-linear functions of $x$ in the plane, can be equivalently represented via complementarity conditions between multipliers and variables $x$. The case of "star-shaped" cells is detailed in [53].

In the case the vector field $f$ is discontinuous on $\Sigma$, a relay representation is possible as follows

$$
\begin{aligned}
\dot{x}(t) \in & \frac{1}{2}\left(A_{1}+A_{2}\right) x(t) \\
& -\frac{1}{2}\left[\left(A_{2}-A_{1}\right) x(t)+a_{2}-a_{1}\right] \operatorname{Sgn}(H x(t)+h) \\
& +\frac{1}{2}\left(a_{1}+a_{2}\right)
\end{aligned}
$$

This relay system does not belong to the class of relay systems studied in Section 7 , however its well-posedness is guaranteed if the switching surface $\Sigma$ is attractive or crossing, using a criterion by Filippov for codimension one switching surfaces (see [40, Section 10]). Notice that if the continuity holds then the right-hand side of (70) is $\frac{1}{2}\left(A_{1}+A_{2}\right) x+v_{1,2}|H x+h|+\frac{1}{2}\left(a_{1}+a_{2}\right)$ for some $v_{1,2}$ from Theorem 4.4. The same process can be done for multiple switching surfaces, but then the uniqueness of solutions may not be assured on codimension $\geq 2$ sliding surfaces (which is the case for the classes of relay systems studied in Section 7). The results in $[53,72]$ also apply in the case where the graphs possess vertical branches, that correspond to a multivalued right-hand side of the evolution problem.

For $n=2$, consider the system (2) with $\left\{d_{j}: j \in \overline{1, p}\right\}$ as in Example 3.1. Under the continuity conditions imposed on the vector field $f(x)=A_{i} x+a_{i}$ if $x \in \chi_{i}, i \in$ $\overline{1, p+1}$, the LCS representation reads as follows

$$
\left\{\begin{aligned}
\dot{x}(t) & =A_{1} x(t)+a_{1}+B_{1} \lambda_{1}+\ldots+B_{p} \lambda_{p} \\
0 & \leq \lambda_{1} \perp H_{1} x+h_{1}+D_{1} \lambda_{1} \geq 0 \\
& \vdots \\
0 & \leq \lambda_{p} \perp H_{p} x+h_{p}+D_{p} \lambda_{p} \geq 0
\end{aligned}\right.
$$

where $D_{j}>0$ and $B_{j} \in \mathcal{M}_{2,1}(\mathbb{R})$ satisfy $\frac{B_{j}}{D_{j}} H_{j}=A_{j}-A_{j+1}$ and $\frac{B_{j}}{D_{j}} h_{j}=a_{j}-a_{j+1}$ for all $j \in \overline{1, p}$.

Further, dropping the continuity conditions, we may embed the system

$$
\dot{x}(t)=A_{i} x+a_{i} \text { if } x \in \chi_{i}, i \in \overline{1, p+1}
$$

into the following relay system

$$
\begin{aligned}
\dot{x}(t) \in A x(t)+a & -\left[B_{1} x(t)+C_{1}\right] \operatorname{Sgn}\left(H_{1} x(t)+h_{1}\right)-\ldots \\
& -\left[B_{p} x(t)+C_{p}\right] \operatorname{Sgn}\left(H_{p} x(t)+h_{p}\right),
\end{aligned}
$$

where $A, B_{j} \in \mathcal{M}_{2,2}(\mathbb{R}), C_{j} \in \mathcal{M}_{2,1}(\mathbb{R})$ for all $j \in \overline{1, p}$ are uniquely determined from the algebraic systems

$$
\begin{gathered}
\left\{\begin{array}{c}
A_{1}=A-B_{1}-B_{2}-\ldots-B_{p} \\
a_{1}=a-C_{1}-C_{2}-\ldots-C_{p}
\end{array}\right. \\
\left\{\begin{array}{c}
A_{2}=A+B_{1}-B_{2}-\ldots-B_{p} \\
a_{2}=a+C_{1}-C_{2}-\ldots-C_{p} \\
\ldots \ldots
\end{array}\right. \\
\left\{\begin{array}{c}
A_{p+1}=A+B_{1}+B_{2}+\ldots+B_{p} \\
a_{p+1}=a+C_{1}+C_{2}+\ldots+C_{p}
\end{array}\right.
\end{gathered}
$$

$\mathrm{RR} \mathrm{n}^{\circ} 7760$ 
(this follows simply from the fact that $\underbrace{\left|\begin{array}{cccc}1 & -1 & \ldots & -1 \\ 1 & 1 & -1 \ldots & -1 \\ \vdots & & & \\ 1 & 1 & \ldots & 1\end{array}\right|}_{p+1}=2^{p} \neq 0$.)

As mentioned in [2] it is possible to construct in a systematic way a relay system from any switching system as in (2), using the functions $\frac{1-\operatorname{sgn}\left(d_{j}(x)\right)}{2}$ and $\frac{1+\operatorname{sgn}\left(d_{j}(x)\right)}{2}$. However, the analysis of the relay systems that arise here (involving products of sign functions) is subtle and deserve more particular attention, despite their very clear definition in the interior of the cells $\chi_{i}$. Finding the class of switching systems such that this "sign formula" provides a well-posed relay system is still largely open.

Under the assumption that for (2) there are $p$ attractive surfaces that generates exactly $m=2^{p}$ different regions and therefore $2^{p}$ vector fields $f_{i}$ 's, the authors of [36] justify a definition of the system on the discontinuity boundaries, starting from a more general nonlinear relay system close to (49) with $\gamma_{i}=0, i=1, \ldots, n$, the constants $k_{l}$ replaced by the functions $f_{l}(x)$ for each $l \in L=\{1, \ldots, m\}$ and $\operatorname{sgn}\left(x_{j}-\theta_{j}^{l}\right)$ replaced by $\operatorname{sgn}\left(d_{j}(x)\right), j=1, \ldots, p$. The main tool in order to identify a selection consistent with the Filippov convexification approach, is to reformulate the multivalued sign function and then to impose the condition that this selection lie in the tangent plane at the discontinuity boundary; this condition is necessary for the sliding motion to occur.

\section{Multimodal systems with multiple criteria}

Let us now turn our attention to a class of switching systems known as piecewiselinear (PWL) systems $[49,48]$. The mechanical system of Example 3.2 belongs to this class. The above models (relay, complementarity systems) cannot be used to prove their well-posedness. PWL systems have been studied in several papers: see [49] for bimodal systems with single criterion and [49], [48], [74] for multimodal systems with multiple criteria. Recently, the study of the well-posedness developed in the above mentioned works was successfully extended by [73] to the more general class of nonlinear systems with multiple modes and multiple criteria. In this section we turn back to the general form of discontinuous system (2). As stressed out from the beginning, the system (2) is not defined on the intersection boundaries $\partial \chi_{i}$. In contrast with the theory of Filippov, in the theory of multimodal systems, to each point on a common boundary of some cells, one associates exactly one of the corresponding vector fields that define the system on a neighborhood of that point. This means that for bimodal systems defined outside the boundary by $f_{1}$ and $f_{2}$, at a point on the discontinuity surface the multivalued part is given by the set of the two vectors, while in the Filippov regularization case, the multivalued part is given by the line segment of ends $f_{1}$ and $f_{2}$. As an example of PWL system, one may consider Example 3.2.

\subsection{The general framework for PWL systems}

In order to give a complete definition of the discontinuous system (2), let us introduce the multifunction $F: \mathbb{R}^{n} \rightarrow \mathbb{R}^{n}$,

$$
F(x)= \begin{cases}\left\{f_{i}(x)\right\} & \text { if } x \in \chi_{i} \\ \bigcup_{i \in I}\left\{f_{i}(x)\right\} & \text { if } x \in \bigcap_{i \in I} \bar{\chi}_{i} \text { for some } I \subseteq \overline{1, m} .\end{cases}
$$

RR $n^{\circ} 7760$ 
The discontinuous system (2) will be restated by the differential inclusion:

$$
\dot{x}(t) \in F(x(t)) .
$$

Definition 13.1. Suppose that there are no left-accumulations of switches. For a given initial state $x(0)=x_{0}$, a function $x:[0, \infty) \rightarrow \mathbb{R}^{n}$ is a solution of the discontinuous system (2) in the sense of Caratheodory, if it is absolutely continuous on each compact subinterval of $[0, \infty)$ and there exists a (measurable) selection $\tilde{f}$ of $F$ such that $x$ and $\tilde{f}$ satisfy the integral equation

$$
x(t)=x_{0}+\int_{0}^{t} \tilde{f}(x(\tau)) d \tau, \quad \forall t \geq 0 .
$$

We note that the above definition extends naturally to the frame of differential inclusions, the concept of a Caratheodory solution for a discontinuous system contained in Definition 5.1.

For $k \geq 1, i \in \overline{1, m}, j \in \overline{1, p}$, we introduce the following notations:

$$
\begin{aligned}
S_{i, j, k} & =\left[d_{j}, L_{f_{i}} d_{j}, \ldots, L_{f_{i}}^{k-1} d_{j}\right]^{T}, \\
T_{i, j, k} & =\left\{\begin{array}{l}
\left\{x \in \mathbb{R}^{n}: S_{i, j, k}(x) \succeq 0\right\}, j \in J_{i}^{1} \\
\left\{x \in \mathbb{R}^{n}: S_{i, j, k}(x) \preceq 0\right\}, j \in J_{i}^{2}
\end{array},\right. \\
T_{i} & =\bigcap_{j \in \overline{1, p}} \bigcap_{k \geq 1} T_{i, j, k}, \\
K_{i, j} & =\left\{x \in \bar{\chi}_{i} \cap \bar{\chi}_{j}: f_{i}(x)=f_{j}(x)\right\}, i \neq j .
\end{aligned}
$$

Remark 13.2. It is easy to verify that for any $i, i_{1}, i_{2}=1$..m one has

$$
\begin{array}{r}
\operatorname{Int}(\chi)_{i}=\chi_{i} \subset T_{i} \subset \bar{\chi}_{i} ; \\
\chi_{i}=\operatorname{Int}\left(T_{i}\right) \text { and } \bar{\chi}_{i}=\bar{T}_{i} ; \\
\bar{T}_{i_{1}} \cap \bar{T}_{i_{2}}=\bar{\chi}_{i_{1}} \cap \bar{\chi}_{i_{2}} .
\end{array}
$$

and if $T_{i_{1}} \cap T_{i_{2}} \neq \emptyset$ then $\left(J_{i_{1}}^{1} \cap J_{i_{2}}^{2} \neq \emptyset\right)$ or $\left(J_{i_{2}}^{1} \cap J_{i_{1}}^{2} \neq \emptyset\right)$.

Necessary and sufficient conditions for the well-posedness of system (2) in the sense of Definition 13.1 have been studied in [73]. In the sequel we give a correct version of Theorem 3.1 in [73]) that wrongly states necessary conditions.

Hypotheses 13.3. $i$ ) For any $M>0$ and each $i \in \overline{1, m}$, there exists $k_{i, M}>0$ such that $f$ verifies the following growth condition

$$
\left\|f_{i}(x)\right\| \leq k_{i, M}(1+\|x\|), \quad \forall x \in \mathbb{R}^{n},\|x\| \leq M .
$$

ii) $f$ is piecewise analytic in the sense that, for any $i \in \overline{1, m}, f_{i}$ is analytic.

Theorem 13.4. Suppose that Hypotheses 13.3 are satisfied. If for any initial condition $x_{0}=x(0)$, the differential inclusion (71) admits a unique solution (in the sense of Caratheodory), then

$$
\begin{aligned}
& \bigcup_{i \in \overline{1, m}} T_{i}=\mathbb{R}^{n}, T_{i} \cap T_{j} \subset K_{i, j} \text { for all } i \neq j \text { and } \\
& T_{i_{1}} \cap T_{i_{2}} \subset \bigcap_{\substack{k \geq 1 \\
j=1 . . p}} \operatorname{Ker}\left(S_{i_{1}, j, k}-S_{i_{2}, j, k}\right) \text { for } i_{1} \neq i_{2} .
\end{aligned}
$$

$\mathrm{RR} \mathrm{n}^{\circ} 7760$ 
Proof. Let $x_{0} \in \mathbb{R}^{n}$ and $x($.$) a solution, with x(0)=x_{0}$. Then $\exists i=1 . . m$ and $\epsilon>0$ such that $x_{0} \in \bar{\chi}_{i}$ and $x(t) \in \bar{\chi}_{i}$ on $[0, \epsilon)$, that is for $t \in[0, \epsilon)$ we have

$$
\begin{gathered}
d_{j}(x(t)) \geq 0 \forall j \in J_{i}^{1} \\
d_{j}(x(t)) \leq 0 \forall j \in J_{i}^{2} .
\end{gathered}
$$

Case 1. If $d_{j}\left(x_{0}\right) \neq 0 \forall j=1 . . p$, then $x_{0} \in \operatorname{Int}\left(T_{i, j, k}\right) \forall k \geq 1$ and $j=1 . . p$, so $x_{0} \in T_{i}$.

Case 2. If there exists $j=1 . . p$ such that $d_{j}\left(x_{0}\right)=0$ and $d_{j}(x(t)) \geq 0$ on $[0, \epsilon)$. Cf. [49], $\exists k_{0} \geq 1$ such that $d_{j}\left(x_{0}\right)=0 \ldots\left(d_{j} \circ x\right)^{k_{0}-1}(0)=0$ and $\left(d_{j} \circ x\right)^{k_{0}}(0)>$ $0 \Leftrightarrow L_{f_{i}}^{k_{0}} d_{j}\left(x_{0}\right)>0$. We obtain $S_{i, j, k} \succcurlyeq 0 \forall k \geq 1$, hence $x_{0} \in T_{i, j, k}$. Similarly, if $d_{j}\left(x_{0}\right)=0$ and $d_{j}(x(t)) \leq 0$ on $[0, \epsilon)$, we get $S_{i, j, k} \preccurlyeq 0 \forall k \geq 1$ and again $x_{0} \in T_{i, j, k}$.

From the above we conclude that $x_{0} \in T_{i}$, so $\bigcup_{i=1 . . m} T_{i}=\mathbb{R}^{n}$. Further, let $x_{0} \in T_{i_{1}} \cap T_{i_{2}}$. We know from Lemma 3.1 in [73] that if $x_{1}$ and $x_{2}$ are two solutions with the same initial condition $x_{0}$, then $x_{1} \equiv x_{2}$ on some interval $[0, \epsilon)$. We obtain that $f_{i_{1}} \equiv f_{i_{2}}$ and $L_{f_{i_{1}}}^{k} d_{j}\left(x_{1}(t)\right)=L_{f_{i_{2}}}^{k} d_{j}\left(x_{2}(t)\right) \forall k \geq 0$ on $[0, \epsilon)$. The last equality implies that $x_{0} \in \operatorname{Ker}\left(S_{i_{1}, j, k}-S_{i_{2}, j, k}\right) \forall k \geq 0$ and $j=1 . . p$.

Remark 13.5. As alluded to in section 5.3, the conditions of Theorem 13.4 guarantee that the switching surfaces are of the crossing type: there are no sliding motions, nor repulsive surfaces. The intuition behind the construction of the sets $T_{i}$ and of $S_{i, j, k}$ is that one observes the way the solutions reach the boundaries, and how they leave them. The solutions may reach and leave the boundaries with various degrees of tangency, as reflected by the calculations of the Lie derivatives that form $S_{i, j, k}$.

As already remarked in [73], the conditions of the above theorem are difficult to check because of the infinite number of intersections in the definition of the sets $T_{i}$. Therefore, the authors of [73] also considered special cases of switched systems, namely systems with single criterion, where the cells $\chi_{i}, i \in \overline{1, m}$ are described by one and only one inequality. The results in [73] extend to nonlinear systems the results in [74] obtained for piecewise linear systems with multiple modes and multiple criteria, described by lexicographic inequalities. In turn, the work in [74] is an extension of papers [49] and [48], where equivalent conditions for the well-posedness of bimodal linear systems with multiple criteria are investigated.

Example 13.6. Let us look at the following system:

$$
\begin{aligned}
& \dot{x}(t)=\left\{\begin{array}{lll}
1 & \text { if } & x(t) \geq 0 \\
-1 & \text { if } & x(t) \leq 0
\end{array}\right. \\
& x(0)=x_{0} .
\end{aligned}
$$

The discontinuity surface is given by $x=0$ and here the multivalued part is $F(x)=$ $\{-1,1\}$. For $\chi_{1}=\mathbb{R}_{+}^{*}$ and $\chi_{2}=\mathbb{R}_{-}^{*}$, we have

$$
\begin{aligned}
& T_{1}=\{x \in \mathbb{R}:[x, 1,0, \ldots, 0] \succeq 0\}=\mathbb{R}_{+} \\
& T_{2}=\{x \in \mathbb{R}:[x, 1,0, \ldots, 0] \preceq 0\}=\mathbb{R}_{-}
\end{aligned}
$$

We have $T_{1} \cap T_{2}=\{0\} \nsubseteq K_{1,2}=\emptyset$, so the above bimodal system does not have a unique Caratheodory solution for any initial point $x_{0}$. In fact, if $x_{0}=0$ the system tends to jump to one of the two possible modes, i.e. there are two solutions: $x(t) \equiv \pm t$. 
Reversing the sign in the above system (see also Example 8), we remark that $T_{1}=$ $\mathbb{R}_{+}^{*}, T_{2}=\mathbb{R}_{-}^{*}$ and $T_{1} \cup T_{2} \neq \mathbb{R}^{2}$, even if $T_{1} \cap T_{2}=\emptyset \subset K_{1,2}$; so Theorem 13.4 above says that the system is not well-posed. In fact we notice that there is no solution starting from 0 , while in the context of Filippov regularization the system has a unique solution for any $x_{0} \in \mathbb{R}$ and the surface $x=0$ is attractive.

Example 13.7. Let us return to Example 3.2 and associate the following bimodal system:

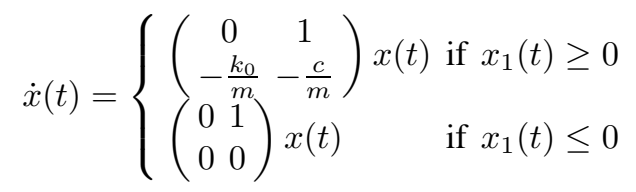

( $k_{0}$ stands for the stiffness). Since $p=1, m=2$, for all $k \geq 3$ we obtain:

$$
\begin{aligned}
S_{1, k}(x) & =\left[x_{1}, x_{2},-\frac{k_{o}}{m} x_{1}-\frac{c}{m} x_{2}, \ldots, c_{k-3}^{1} x_{1}+c_{k-3}^{2} x_{2}\right], \\
S_{2, k}(x) & =\left[x_{1}, x_{2}, 0, \ldots, 0\right], \\
T_{1, k} & =\left\{\left(x_{1}, x_{2}\right): x_{1}>0 \vee x_{1}=0, x_{2} \geq 0\right\}, \\
T_{2, k} & =\left\{\left(x_{1}, x_{2}\right): x_{1}<0 \vee x_{1}=0, x_{2} \leq 0\right\},
\end{aligned}
$$

where $c_{k-3}^{1}, c_{k-3}^{2} \in \mathbb{R}$ are some constants. Then $T_{1} \cap T_{2}=\{(0,0)\} \subset K_{1,2}=\{x \in$ $\left.\mathbb{R}^{2}: k_{0} x_{1}+c x_{2}=0\right\}$ and the necessary condition of Theorem 13.4 is verified.

The well-posedness of the mass spring/dashpot system as in Example 3.2 is provided in [62] where it is shown that $q$ is $\mathcal{C}^{1}, \dot{q}$ is absolutely continuous and $\ddot{q}$ exists almost everywhere. The model used in [62] assumes that the damping term takes the value 0 at $q=0$.

\subsection{A particular case of PWL system}

Since it is the purpose of our work to identify common subclasses between various types of switching systems, let us consider a subclass of the above PWL systems, that allows us to make a link with relay systems. Let $f_{0}: \mathbb{R}^{n} \rightarrow \mathbb{R}^{n}$ be a given function and $B \in \mathcal{M}_{n, p}(\mathbb{R})$. In the cells $\left(\chi_{i}\right)_{i \in \overline{1, m}}$, we consider the following discontinuous system

$$
\dot{x}(t)=f_{0}(x(t))+\lambda_{i} \text { if } x(t) \in \chi_{i},
$$

where $\lambda_{i}=-\sum_{j \in J_{i}^{1}} B_{\bullet} j+\sum_{j \in J_{i}^{2}} B_{\bullet}, \lambda_{i}^{T} \in \mathbb{R}^{n}$.

Since on the intersection boundaries between $\bar{\chi}_{i}, i \in \overline{1, m}$ the system is not yet defined, we shall consider two different definitions of the discontinuous system $\dot{x}(t)=$ $f(x(t))$ on these boundaries, in order to have a good definition of the system on the whole space $\mathbb{R}^{n}$. Different definitions may be considered on the boundaries.

I. The first approach deals with the possibility for the discontinuous vector field $f$ to take, at every point on the intersection boundaries, any value from the set of all values of the vector fields that define the system on a neighborhood of this point; this definition allows one to settle a necessary and sufficient condition in order to have a unique smooth continuation from any initial state and so, the well-posedness of the discontinuous system (74).

Let $f_{i}: \bar{\chi}_{i} \rightarrow \mathbb{R}^{n}, f_{i}(x)=f_{0}(x)+\lambda_{i}, i \in \overline{1, m}$ and suppose that $f_{0}$ is an analytic function that satisfies Hypotheses 13.3, i) with $f_{i}$ replaced by $f_{0}$. Then, Theorem 13.4 can be applied to the problem (74) and the well-posedness is straightforward. 
In what follows we present a corollary of Theorem 13.4 which may also be regarded as an extension to affine systems of the results in [74]. Let $A \in \mathcal{M}_{n, n}(R)$ be a given matrix and let us consider in the cells $\left(\chi_{i}\right)_{i \in \overline{1, m}}$ a discontinuous affine system defined as follows

$$
\dot{x}(t)=A x(t)+\lambda_{i} \quad \text { if } x(t) \in \chi_{i} .
$$

Due to the particular form of $d_{j}$ and $f_{i}$ we have

$$
L_{f_{i}}^{k} d_{j}(x)=H_{j} \bullet A^{k-1} f_{i}(x) \text { for all } k \geq 1 .
$$

For $i \in \overline{1, m}, j \in \overline{1, p}$, let us adopt the following notations:

$$
\begin{aligned}
S_{i, j} & =\left[d_{j}, L_{f_{i}} d_{j}, \ldots, L_{f_{i}}^{k_{j}-1} d_{j}\right]^{T}, \\
T_{i, j} & =\left\{\begin{array}{ll}
\left\{x \in \mathbb{R}^{n}:\right. & \left.S_{i, j}(x) \succeq 0\right\}, j \in J_{i}^{1} \\
\left\{x \in \mathbb{R}^{n}:\right. & \left.S_{i, j}(x) \preceq 0\right\}, j \in J_{i}^{2}
\end{array},\right. \\
T_{i} & =\bigcap_{j \in \overline{1, p}} T_{i, j},
\end{aligned}
$$

where $k_{j}$ is the maximal integer value $\left(k_{j} \leq n+1\right)$ such that the matrix $\left[H_{j \bullet}^{T},\left(H_{j} \bullet A\right)^{T}\right.$, $\left.\ldots,\left(H_{j} A^{k_{j}-2}\right)^{T}\right]^{T}$ has a row-full rank (in particular, this holds if $\left(H_{j \bullet} A\right)$ is observable).

We remark here that $f_{i}$ and $d_{j}$ are analytic, $\forall i \in \overline{1, m}$ and $j \in \overline{1, p}$. Moreover, by taking

$$
M=\max \left\{\|A\|_{1}, \sum_{j \in \overline{1, p}}\left\|B_{\bullet}\right\|_{1}\right\},
$$

we find that for any $i \in \overline{1, m}, f_{i}$ satisfies the linear growth condition

$$
\left\|f_{i}(x)\right\|_{1} \leq\|A\|_{1}\|x\|_{1}+\sum_{j \in \overline{1, p}}\left\|B_{\bullet}\right\| \leq M\left(1+\|x\|_{1}\right),
$$

where $\|A\|_{1}$ stands for the matrix norm (the maximum absolute column sum).

The particular description of the cells (Section 2.1) together with the above definition of the discontinuous vector field $f$ along $\bar{\chi}_{i}$ allows one to derive an equivalent condition for the well-posedness of the discontinuous system which is easier to verify (the definition of $T_{i}$ is given in terms of finite intersections). This is done in the next proposition.

Proposition 13.8. For any initial condition $x_{0}=x(0)$, the discontinuous system (75) admits a unique solution (in the sense of Caratheodory) if and only if

$$
\bigcup_{i \in \overline{1, m}} T_{i}=\mathbb{R}^{n} \text { and } T_{i} \cap T_{j} \subset K_{i, j} \text { for all } i \neq j .
$$

Proof. It is easy to see that for any $i \in \overline{1, m}$ and $j \in J_{i}^{1}$, the set $T_{i, j}$ may be written as an infinite intersection as follows

$$
\begin{aligned}
& T_{i, j}=\left\{x \in \mathbb{R}^{n} ; S_{i, j}(x) \succeq 0\right\} \\
& =\bigcap_{k \geq 2}\left\{x \in \mathbb{R}^{n}:\left(d_{j}(x), H_{j \bullet} f_{i}(x), \ldots, H_{j} A^{k-2} f_{i}(x)\right)^{T} \succeq 0\right\},
\end{aligned}
$$

$\mathrm{RR} \mathrm{n}^{\circ} 7760$ 
that is $T_{i, j}$ is the limit of a decreasing sequence of sets. Indeed, the inclusion " $\supseteq$ " is obvious, while for the other one, it is sufficient to observe that $S_{i, j}(x)=0$ (componentwise) implies, by the choice of $k_{j}$, that $H_{j} \bullet A^{k} f_{i}(x)=0$ for any $k \geq k_{j}+2$. Similar arguments work for $T_{i, j}$ with $j \in J_{i}^{2}$.

II. The second approach yields a sufficient condition for the well-posedness of the system (2). Employing the standard multivalued Sign function $\left(\left|\alpha_{i}\right|=\left|\beta_{i}\right|=1\right.$ in Figure 2 (a)), we embed (74) into the following differential inclusion:

$$
\dot{x}(t) \in f_{0}(x(t))-B \operatorname{Sgn}(d(x(t)))
$$

where $\operatorname{Sgn}(d(x))=\left(\operatorname{Sgn}\left(d_{1}(x)\right), \ldots, \operatorname{Sgn}\left(d_{p}(x)\right)\right)^{T}$. Clearly, for $x \in \chi_{i}$, the righthand side in (77) is exactly $f_{i}(x)$. If $x \in \bigcap_{i \in I} \bar{\chi}_{i}$ for some $I \subseteq \overline{1, m}$ and

$$
J(x)=\left\{j \in \overline{1, p}: d_{j}(x)=0\right\},
$$

the right-hand side in (77) becomes

$$
f_{0}(x)-\sum_{j \notin J(x)} B_{\bullet} \operatorname{Sgn}\left(d_{j}(x)\right)+\sum_{j \in J(x)} B_{\bullet}[-1,1] .
$$

Now, by (77), the system (2) is well-defined on the whole state space.

Hypotheses 13.9. a) The function $f_{0}$ is Lipschitz continuous, that is there exists $L>0$ such that

$$
\left\|f_{0}(x)-f_{0}(y)\right\| \leq L\|x-y\|, \quad \forall x, y \in \mathbb{R}^{n} .
$$

b) There exists a symmetric positive definite matrix $P \in \mathcal{M}_{n, n}(\mathbb{R})$ such that $P B=C^{T}$ for all $i \in \overline{1, n}$.

The next result is an application of Theorem 7.11 to the particular case of differential inclusions considered in (74).

Proposition 13.10. Consider inclusion (77) and assume that Hypotheses 13.9 are satisfied. Then, for any initial condition $x_{0} \in \mathbb{R}^{n}$, the differential inclusion (77) has a unique Lipschitz solution with essential bounded derivative.

Remark 13.11. We emphasize here that in both of these approaches, the employed solution concept is that of a Caratheodory solution for a differential inclusion (see Definition 7.10). However, in the first approach, taking into account the conditions to be verified for the existence and uniqueness of a Caratheodory solution, supplementary assumption should be required namely, the non existence of right-accumulation of the switches.

\section{Numerical computation of the solutions}

The numerical simulation of nonsmooth dynamical systems (mechanical systems with impact and friction, electrical circuits with ideal components) is a vast field of investigation $[2,3,4]$. Two major methods exist for the simulation of dynamical systems with nonsmooth events: time-stepping (or event-capturing) schemes, and event-driven 
schemes (see e.g. [2, pp.199-201] for a definition). Let us focus on time-stepping schemes. The AVS in (12)-(13) is discretized as:

$$
\left\{\begin{array}{l}
x_{k+1}=x_{k}+h g\left(x_{k+1}\right)+h B \lambda_{k+1} \\
y_{k+1}=C x_{k+1}+c+D \lambda_{k+1} \\
\left(s-\lambda_{k+1}\right)^{T} y_{k+1} \geq 0, \quad \forall s \in K
\end{array}\right.
$$

where $h>0$ is the time step. The last line is equivalent to $-y_{k+1} \in N_{K}\left(\lambda_{k+1}\right) \Leftrightarrow$ $\lambda_{k+1} \in \partial \psi_{K}^{*}\left(-y_{k+1}\right)$. Therefore the discrete-time system (78) can be rewritten equivalently in different ways depending on the data (mainly $D$ and $K$ ), still using the convex analysis tools as in the above examples. We retrieve here that the operator $x_{k+1} \mapsto\left(D \cdot+N_{K}\right)^{-1}\left(-C x_{k+1}-c\right)$ plays a central role in (78), which can be rewritten compactly as:

$$
x_{k+1}=x_{k}+h g\left(x_{k+1}\right)+h B\left(D \cdot+N_{K}\right)^{-1}\left(-C x_{k+1}-c\right)
$$

that is a generalized equation with unknown $x_{k+1}$ to be solved to advance the scheme from step $k$ to step $k+1$.

It is noteworthy that in practice one often chooses a more general discretization such as $\theta$-methods $[2,61]$. The convergence of Euler-like implicit time-stepping methods has been shown in [20] for LCS and for linear relay systems in [46], see also [61, Sections 7 and 8 ] in the more general setting of differential variational inequalities, and [45] for generalizations of [20]. The main assumptions in [20] and [46] are made on $D$ and on the existence of solutions to (79), which is an LCP for discretized LCS. The discretized differential inclusion (34) satisfying the conditions of Theorem 7.11 is studied in [1]. The results of [9] can then straightforwardly be used to prove the convergence of the implicit Euler method, with order $\frac{1}{2}$. Most interestingly it is shown in [1] that the implicit method, contrarily to the explicit one [75], can numerically stabilize the discrete solution on the sliding surfaces in a smooth way, without spurious numerical oscillations (despite both the implicit and the explicit method converge, their qualitative behaviour on sliding surfaces is quite different, see the simulation results in [1] and [75]). Finally let us mention the works [70,69] in which a specific description of the cells $\chi_{i}$ is made, that allows one to derive accurate event-driven schemes. The advantage of event-driven schemes over time-stepping ones, is that they may allow for higher accuracy. However they are also prone to "epsilon-tuning" process due to the necessity to incorporate higher-order derivatives estimations when the trajectories attain, or lie on boundary surfaces. This is often quite a burden in the numerical implementation. Moreover they cannot be implemented (except if the solution is known in advance!) when accumulations of events exist.

\section{Conclusions}

This paper presents a brief introduction to switching systems, their well-posedness and their relationships with relay and complementarity dynamical systems, as summarized in Figure 1. The Filippov's regularization allows one to embed switching systems into a general framework of differential inclusions with absolutely continuous solutions. This however is often not sufficient to prove the uniqueness of the solutions, to derive good numerical algorithms, and more compact formalisms lend themselves much better to deep mathematical and numerical analysis. This is why relay and complementarity systems offer a very attractive point of view, despite they represent only 
narrow classes of switching systems from the point of view of the cells topology. Dissipativity, the $P$ property of matrices and maximal monotonicity of operators appear to be essential tools. From the point of view of applications, they however represent large and important classes of systems: mechanical systems with piecewise linear interface laws (impacts, friction), electrical circuits with piecewise linear components (ideal diodes, switches), feedback systems with relay and discontinuous controllers, genetic regulatory networks etc. It may even be said that from the applications point of view, switching systems as in (2) model only a narrow class of mechanical and electrical systems, which often possess a positive relative degree and solutions that have to be sought in distribution spaces. Convexity appears to be a central feature for all mathematical formalisms considered (convexity of the cells of the switching systems, convexity of the sets that define the underlying variational inequality constraint for relay and complementarity systems). Whether all this material extends to non convex cases (considering for instance the so-called prox-regular sets which are an interesting extension of convex sets $[16,25])$ may be a topic of interest. Finally we focus on discontinuous systems with continuous solutions. Indeed including state jumps requires to reconsider all the mathematical formalisms (one then has to work with measure or distribution differential inclusions), and this is beyond the scope of this paper.

Remark 15.1. Dedicated software for the class of set-valued, nonsmooth dynamical systems studied in this paper and based on the above time-stepping schemes, are not widely developed. Let us mention the SICONOS platform, an open-source software developed at INRIA [1, 3, SICONOS].

\section{References}

[1] V. Acary, B. Brogliato (2010). Implicit numerical scheme and chattering-free implementation of sliding mode systems. Systems and Control Letters, 59(5), 284-293.

[2] V. Acary, B. Brogliato (2008). Numerical Methods for Nonsmooth Dynamical Systems. Springer Verlag Berlin Heidelberg, Lecture Notes in Applied and Computational Mechanics, vol.35.

[3] V. Acary, O. Bonnefon, B. Brogliato (2011). Nonsmooth Modeling and Simulation for Switched Circuits. Springer Verlag Berlin Heidelberg, Lecture Notes in Electrical Engineering, vol.69.

[4] V. Acary, O. Bonnefon, B. Brogliato (2010). Time-stepping numerical simulation of switched circuits within the nonsmooth dynamical systems approach. IEEE Transactions on Computer-Aided Design of Integrated Circuits and Systems, 29(7),1042-1055.

[5] V. Acary, B. Brogliato (2004). Coefficients de restitution et efforts aux impacts. Revue et comparaison des estimations analytiques. INRIA Research Report 5401, December 2004, available at http://hal.inria.fr .

[6] V. Acary, B. Brogliato, D. Goeleven (2008). Higher order Moreau's sweeping process: mathematical formulation and numerical simulation. Mathematical Programming A, 113(1), 133-217. 
[7] P. Ballard (2000). The dynamics of discrete mechanical systems with perfect unilateral constraints. Archive for Rational Mechanics and Analysis, 154(3), 199274.

[8] J. Bastien and C.H. Lamarque (2008). Persoz's gephyroidal model described by a maximal monotone differential inclusion. Arch. Appl. Mech., 78, 393-407.

[9] J. Bastien, M. Schatzman (2002). Numerical precision for differential inclusions with uniqueness. Mathematical Modelling and Numerical Analysis (ESAIM:M2AN), 36(3), 427-460.

[10] M. di Bernardo, C.J. Budd, A.R. Champneys, P. Kowalczyk (2007). Piecewisesmooth Dynamical Systems. Theory and Applications. Springer, Applied Mathematical Sciences, vol.163.

[11] B. Brogliato (1999). Nonsmooth Mechanics. Springer Verlag London, Communications and Control Engineering, (2nd ed.).

[12] B. Brogliato (2003). Some perspectives on the analysis and control of complementarity systems. IEEE Transactions on Automatic Control, 48(6), 918-935.

[13] B. Brogliato (2004). Absolute stability and the Lagrange-Dirichlet theorem with monotone multivalued mappings. Systems and Control Letters, 51(5), 343-353.

[14] B. Brogliato, A. Daniilidis, C. Lemaréchal, V. Acary (2006). On the equivalence between complementarity systems, projected systems and differential inclusions. Systems and Control Letters, 55(1), 45-51.

[15] B. Brogliato, R. Lozano, B. Maschke, O. Egeland (2007). Dissipative Systems Analysis and Control. Theory and Applications. Springer Verlag London, Communications and Control Engineering, (2nd ed.)

[16] B. Brogliato, L. Thibault (2010). Existence and uniqueness of solutions for nonautonomous complementarity dynamical systems. Journal of Convex Analysis, 17(3-4), 961-990.

[17] B. Brogliato, D. Goeleven (2011). Well-posedness, stability and invariance results for a class of multivalued Lur'e dynamical systems. Nonlinear Analysis. Theory, Methods and Applications, 74(1), 195-212.

[18] B. Brogliato, W.P.M.H. Heemels (2009). Observer design for Lur'e systems with multivalued mappings: a passivity approach. IEEE Transactions on Automatic Control, 54(8), 1996-2001, August.

[19] M.K. Çamlibel, W.P.M.H. Heemels, J.M. Schumacher (2002). On linear passive complementarity systems. European Journal of Control, 8(3), 220-237.

[20] M.K. Çamlibel, W.P.M.H. Heemels, J.M. Schumacher (2002). Consistency of a time-stepping method for a class of piecewise linear networks. IEEE Transactions on Circuits and Systems, I: Fundamental Theory and Applications, 49(3), 349357.

[21] M.K. Çamlibel, J.-S. Pang, J. Shen (2006). Lyapunov stability of complementarity and extended systems. SIAM J. Control Optim., 17(4), 1056-1101. 
[22] M.K. Çamlibel, J.-S. Pang, J. Shen (2006). Conewise linear systems: nonzenoness and observability. SIAM J. Control Optim., 45(5), 1769-1800.

[23] R. Casey, H. de Jong, J.L. Gouzé (2006). Piecewise linear models of genetic regulatory networks: Equilibria and their stability. Journal of Mathematical Biology, 52(1), 27-56.

[24] S.L. Chen, K.K. Tan, S. Huang (2011). Identification of Coulomb friction impeded systems with a triple relay feedback apparatus. IEEE Transactions on Control Systems Technology, in press.

[25] G. Colombo, L. Thibault (2010). Prox-regular sets and applications. in Handbook of Nonconvex Analysis, D.Y. Gao, D. Motreanu eds., International Press (2010), ISBN: 978-1-57146-200-8.

[26] J. Cortes (2008). Discontinuous dynamical systems. A tutorial on solutions, nonsmooth analysis, and stability. IEEE Control Systems Magazine, 36-73.

[27] R.W. Cottle, J.S. Pang, R.E. Stone (1992). The Linear Complementarity Problem. Academic Press.

[28] A.A. ten Dam, K.F. Dwarshuis, J.C. Willems (1997). The contact problem for linear continuous-time dynamical systems: a geometric approach. IEEE Transactions on Automatic Control, 42(4), 458-472.

[29] M.-F. Danca (2001). On a class of discontinuous dynamical systems. Mathematical Notes, 2(2), 103-116.

[30] M.-F. Danca (2007). On a class of non-smooth dynamical systems: a sufficient condition for smooth versus non-smooth solutions. Regular and Chaotic Dynamics, 12(1), 1-11.

[31] M.-F. Danca (2010). On the uniqueness of solutions to a class of discontinuous dynamical systems. Nonlinear Analysis: Real World Applications, 11(3), 14021412 .

[32] H. de Jong (2002). Modeling and simulation of genetic regulatory systems: a literature review. Journal of Computational Biology, 9(1), 67-103.

[33] H. de Jong, J.-L. Gouzé, C. Hernandez, M. Page, T. Sari, J. Geiselmann (2004). Qualitative simulation of genetic regulatory networks using piecewise-linear models. Bull. Math. Biol., 66(2), 301-340.

[34] M. di Bernardo, C. Budd, A.R. Champneys, P. Kowalczyk (2008). Piecewisesmooth Dynamical Systems: Theory and Applications. Springer Verlag, Applied Mathematical Sciences, London.

[35] M. di Bernardo, U. Montanaro, S. Santini (2010). Minimal control synthesis adaptive control of continuous bimodal piecewise affine systems. SIAM J. Control Optim., 48(7), 4242-4261.

[36] L. Dieci and L. Lopez (2011) Sliding motion on discontinuity sufaces of high co-dimension. A construction for selecting a Filippov vector field. Numerische Mathematik, 117(4), 779-811. 
[37] L. Dieci and L. Lopez (2009). Sliding motion in Flippov differential systems: Theoretical results and a computational approach. SIAM J. on Numerical Analysis, 47(3), 2023-2051.

[38] F. Facchinei, J.-S. Pang (2002). Finite-Dimensional Variational Inequalities and Complementarity Problems, Springer.

[39] E. Farcot and J.L. Gouzé (2008). A mathematical framework for the control of piecewise-affine models of gene networks. Automatica, 44(9), 2326-2332.

[40] A.F. Filippov (1988). Differential Equations with Discontinuous Righthand Sides, Kluwer Academic Publishers, Dordrecht.

[41] R. Frasca, M.K. Camlibel, I.C. Goknar, L. Iannelli, F. Vasca (2010) Linear passive networks with ideal switches: consistent initial conditions and state discontinuities. IEEE Transactions on Circuits and Systems-I: Regular Papers, 57(12), $3138-3151$.

[42] C. Glocker (2001). Set-valued Force Laws: Dynamics of Non-Smooth Systems. Springer, Lecture Notes in Applied Mechanics, vol.1.

[43] J.L. Gouzé, T. Sari (2002). A class of piecewise linear differential equations arising in biological models. Dynamical Systems: An International Journal, 17(4), 299-316.

[45] L. Han, A. Tiwari, M.K. Çamlibel, J.S. Pang (2009). Convergence of timestepping schemes for passive and extended linear complementarity systems. SIAM J. on Numerical Analysis, 47(5), 3768-3796.

[46] W.P.M.H. Heemels, M.K. Çamlibel, J.M. Schumacher (2000). A time-stepping method for relay systems. Proceedings of the 39th IEEE Conference on Decision and Control, Sydney, Australia, 4461-4466.

[47] W.P.M.H. Heemels, M.K. Çamlibel, J.M. Schumacher, B. Brogliato (2010). Observer-based control of linear complementarity systems International Journal of Robust and Nonlinear Control, 21(10), 1193-1218.

[48] J. Imura (2003). Well-posedness analysis of switch-driven piecewise affine systems. IEEE Transactions on Automatic Control, 48(11), 1926-1935.

[49] J. Imura, A. van der Schaft (2000). Characterization of well-posedness of piecewise affine systems. IEEE Transactions on Automatic Control, 45(9), 1600-1619.

[50] M. Johansson (2003). Piecewise Linear Control Systems. Springer-Verlag Berlin Heidelberg.

[51] A. Jokic, M. Lazar, P.P.J. van den Bosch (2009). On constrained steady-state regulation : dynamic KKT controllers. IEEE Transactions on Automatic Control, 54(9), 2250-2254.

[52] T. Kato (1970). Accretive operators and nonlinear evolution equations in Banach spaces. Nonlinear Functional Analysis, Proceedings of Symposia in Pure Mathematics, 18 Part 1, 138-161. 
[53] D.M.W. Leenaerts, W.M.G. van Bokhoven (1998). Piecewise Linear Modeling and Analysis. Kluwer Academic Publishers, Boston/Dordrecht/London.

[54] C. Lin, Q-G. Wang (2002). On uniqueness of solutions to relay feedback systems. Automatica, 38(1), 177-180.

[55] Y.J. Lootsma, A.J. van der Schaft, M.K. Çamlibel (1999). Uniqueness of solutions of relay systems. Automatica, 35(3), 467-478.

[56] A. Machina, A. Ponosov (2011). Filippov solutions in the analysis of piecewise linear models describing gene regulatory networks. Nonlinear Analysis: Theory, Methods and Applications, 74, 882-900.

[57] M.D.P. Monteiro Marques (1993). Differential Inclusions in Nonsmooth Mechanical Problems: Shocks and Dry Friction. Birkhauser Basel, Progress in Nonlinear Differential Equations and their Applications, vol.9.

[58] J.J. Moreau (1977). Evolution problem associated with a moving convex set in a Hilbert space. Journal of Differential Equations, 26, 347-374.

[59] E. Moulay and W. Perruquetti (2006). Finite-time stability and stabilization: State of the art. in Advances in Variable Structure and Sliing mode Control, Lecture Notes in Control and Information Sciences, vol.334, 23-41.

[60] Y.V. Orlov (2009). Discontinuous Systems. Lyapunov Analysis and Robust Synthesis under Uncertainty Conditions. Springer Verlag, Communications and Control Engineering, London.

[61] J.S. Pang, D. Stewart (2008). Differential variational inequalities. Mathematical Programming A, 113(2), 345-424.

[62] L. Paoli, M. Schatzman (1993). Mouvement à un nombre fini de degrés de liberté avec contraintes unilatérales : cas avec perte d'énergie. Modèl. Math. Anal. Numér. (M2AN), 27(6), 673-717.

[63] A. Pavlov, N. van de Wouw, H. Nijmejer (2005). Uniform Output Regulation of Nonlinear Systems: A Convergent Dynamics Approach. Birkhauser, Boston.

[64] E. Plahte, S. KjØglum (2005). Analysis and generic properties of gene regulatory networks with graded response functions. Physica D, 201, 150-176.

[65] A.Yu. Pogromsky, W.P.M.H. Heemels, H. Nijmejer (2003). On solution concepts and well-posedness of linear relay systems. Automatica, 39(12), 2139-2147.

[66] R.T. Rockafellar (1973). Convex Analysis. Princeton University Press.

[67] S. Scholtes (1994). Introduction to Piecewise Differentiable Equations. Habilitation thesis, Institut für Statistik und Mathematische Wirtschaftstheorie, Universität Karlsruhe.

[SICONOS] http://siconos.gforge.inria.fr/

[69] D. Stewart (1990). A high accuracy method for solving ODEs with discontinuous right-hand side. Numerische Mathematik, 58, 299-328. 
[70] D. Stewart (1992). Numerical methods for friction problems with multile contacts. J. Austral. Math. Soc. Ser. B, 34, 212-228.

[71] L.Q. Thuan and M.K. Çamlibel (2011). Continuous piecewise affine dynamical systems do not exhibit Zeno behaviour. IEEE Transactions on Automatic Control, in press.

[72] L. Vandenberghe, B.L. de Moor, J. Vandewalle (1989). The generalized linear complementarity problem applied to the complete analysis of resistive piecewiselinear circuits. IEEE Transactions on Circuits and Systems, 36, 1382-1391.

[73] C.Z. Wu, K.L. Teo, V. Rehbock, G.G. Liu (2009). Existence and uniqueness of solutions of piecewise nonlinear systems. Nonlinear Analysis: Theory, Methods and Applications, 71(12), 6109-6115.

[74] X. Xia (2002). Well posedness of piecewise-linear systems with multiple modes and multiple criteria. IEEE Trans. Autom. Control, 47(10), 1716-1720.

[75] X. Yu, B. Wang, Z. Galias, G. Chen (2007). Discretization effect on equivalent control-based multi-input sliding-mode control systems. IEEE Transactions on Automatic Control, 53(6), 1563-1569.

[76] J. Zhao, D.J. Hill (2007). Dissipativity theory for switched systems. IEEE Transactions on Automatic Control, 53(4), 941-953. 
Centre de recherche INRIA Grenoble - Rhône-Alpes 655, avenue de l'Europe - 38334 Montbonnot Saint-Ismier (France)

Centre de recherche INRIA Bordeaux - Sud Ouest : Domaine Universitaire - 351, cours de la Libération - 33405 Talence Cedex Centre de recherche INRIA Lille - Nord Europe : Parc Scientifique de la Haute Borne - 40, avenue Halley - 59650 Villeneuve d'Ascq Centre de recherche INRIA Nancy - Grand Est : LORIA, Technopôle de Nancy-Brabois - Campus scientifique 615, rue du Jardin Botanique - BP 101 - 54602 Villers-lès-Nancy Cedex

Centre de recherche INRIA Paris - Rocquencourt : Domaine de Voluceau - Rocquencourt - BP 105 - 78153 Le Chesnay Cedex

Centre de recherche INRIA Rennes - Bretagne Atlantique : IRISA, Campus universitaire de Beaulieu - 35042 Rennes Cedex

Centre de recherche INRIA Saclay - Île-de-France : Parc Orsay Université - ZAC des Vignes : 4, rue Jacques Monod - 91893 Orsay Cedex

Centre de recherche INRIA Sophia Antipolis - Méditerranée : 2004, route des Lucioles - BP 93 - 06902 Sophia Antipolis Cedex 